\title{
MULTIGRID ANALYSIS OF FINITE ELEMENT METHODS WITH NUMERICAL INTEGRATION
}

\author{
CHARLES I. GOLDSTEIN
}

\begin{abstract}
We analyze multigrid convergence rates when elliptic boundary value problems are discretized using finite element methods with numerical integration. The resulting discrete problem does not fall into the standard variational framework for analyzing multigrid methods since the bilinear forms on different grid levels are not suitably related to each other. We first discuss extensions of the standard variational multigrid theory and then apply these results to the case of numerical quadrature. In particular, it is shown that the $\mathscr{V}$ cycle algorithm has a convergence rate independent of grid size under suitable conditions.
\end{abstract}

\section{INTRODUCTION}

Multigrid methods provide a very powerful tool for solving the system of equations resulting from the discretization of elliptic boundary value problems in two or three dimensions. We refer to [1-3] and references cited there for comprehensive treatments of various multigrid methods. There has been a great deal of research devoted to an analysis of the convergence properties of these methods. One approach, based on Fourier analysis, is applicable to a rather limited number of situations (see, e.g., [1, 2, 4, 5]). A more general approach is based on a variational formulation of multigrid (see [2, 3, 6-12] and references cited therein). This variational framework includes the system of equations resulting from finite element discretizations in general domains.

In this paper, we analyze the convergence of multigrid methods for symmetric uniformly elliptic boundary value problems when numerical quadrature is used to approximately evaluate the finite element stiffness matrix (see [13-15]). Numerical integration is often important for the practical implementation of finite element methods and is commonly present in finite element computer codes. (Furthermore, many finite difference schemes can be obtained using a finite element method with numerical quadrature.) When numerial quadrature is used, the resulting discrete problem does not fall into the variational framework cited above, since the bilinear forms on different grid levels are not suitably related to each other (e.g., condition (A.2) in $\$ 2$ fails to hold).

Received August 29, 1989; revised May 29, 1990.

1980 Mathematics Subject Classification (1985 Revision). Primary 65F10, 65N30.

This work was supported by the Applied Mathematical Sciences program of the U. S. Department of Energy under Contract No. DE-AC02-76CH00016. 
An extension of the standard variational multigrid framework was recently provided in [16]. Results in [16] as well as additional results proved in this paper are employed in $\S 4$ to obtain multigrid estimates for the finite element method with numerical quadrature. Note that an abstract multigrid framework, such as that discussed in $\S 3$ below, can be very useful in connection with various other "variational crimes" that occur in connection with practical implementations of finite element and multigrid methods. Some examples of this are presented and analyzed in [16]. We also refer to [17] for a multigrid analysis of some nonconforming finite element methods for which the sequence of grids is not nested and (A.2) does not hold.

We now outline the remainder of the paper. In $\S 2$, we briefly describe a variational formulation of the multigrid method and state some known convergence results. We restrict our discussion throughout this paper to the standard $\mathscr{V}$ cycle (i.e., the same number of smoothings is used on all grid levels) and variable $\mathscr{V}$ cycle (i.e., the number of smoothings is increased geometrically on coarser levels), although analogs of many of the results hold for the $\mathscr{W}$ cycle as well. We consider both the symmetric and nonsymmetric multigrid operators, $B_{J}^{S}$ and $B_{J}^{N}$, respectively, where $J+1$ is the number of grid levels. $B_{J}^{S}$ corresponds to the multigrid algorithm for which the number of presmoothings (i.e., the smoothings occurring before the coarse grid correction) is equal to the number of postsmoothings on each grid level. $B_{J}^{N}$ corresponds to the algorithm for which there is no postsmoothing on any grid level.

Some of the assumptions in $\S 2$ fail to hold when numerical quadrature is used and for many other practical situations. In $\S 3.1$, we state some results proved in [16] for the symmetric operator $B_{J}^{S}$ under weaker assumptions than in $\S 2$. Additional results are stated in $\S 3.2$ that hold for both $B_{J}^{N}$ and $B_{J}^{S}$ under suitable assumptions. These results are proved in the Appendix.

The main results are contained in $\S 4$, where we use the results in $\S 3$ to analyze the multigrid method when a sufficiently accurate quadrature scheme is used to compute the finite element stiffness matrix (see (4.5)). An accuracy condition of this kind is also required for the finite element error analysis of numerical integration and is referred to as the "patch test" in $[13,14]$. It is proved in $\S 4.1$, using methods and results from this finite element analysis, that the assumptions in $\S 3$ are satisfied. In Theorem 4.2, we provide bounds on the condition number of $B_{J}^{S} A_{J}$, indicating that $B_{J}^{S}$ can be used effectively as a preconditioner even with only one presmoothing on all grid levels, where $A_{J}$ is the discrete elliptic operator. It is proved in Theorems $4.3 \mathrm{~S}$ and $4.3 \mathrm{~N}$ for $B_{J}=B_{J}^{S}$ and $B_{J}=$ $B_{J}^{N}$, respectively, that $I-B_{J} A_{J}$ is a contraction operator with contraction number independent of $J$ provided suitable assumptions hold. For the variable $\mathscr{V}$ cycle it is assumed that $h^{\sigma_{1}} m^{-\sigma_{2}}$ is sufficiently small and shown that the contraction number is bounded by $\delta+O\left(h^{\sigma_{1}} m^{-\sigma_{2}}\right)$, where $\delta$ is the contraction number without numerical integration, $h=h_{J}$ is the grid size, $m$ is the number of presmoothings on the finest grid level, and $\sigma_{1}$ and $\sigma_{2}$ are positive constants 
that depend on the accuracy of the quadrature scheme. An analogous result is proved for the standard $\mathscr{V}$ cycle, except that $h$ is replaced by the grid size on the coarsest grid level on which the stiffness matrix is not computed exactly.

\section{MULTIGRID ALGORITHMS}

In this section, we briefly describe the multigrid method using the variational framework in [9] and then state some known convergence results (see Theorem 2.1). We refer to $[2,3,6-12]$ for detailed treatments of variational multigrid methods.

2.1. Definitions and assumptions. Suppose we have a sequence of finite-dimensional vector spaces, $M_{0}, M_{1}, \ldots, M_{J}$, and corresponding linear operators $I_{j}$ : $M_{j-1} \rightarrow M_{j}, 1 \leq j \leq J$. Furthermore, suppose we are given positive definite symmetric bilinear forms $A_{j}($,$) and (,)_{j}$, defined on $M_{j} \times M_{j}$ for each $j$ with corresponding norms denoted by \|\|$_{A_{j}}$ and \|\|$_{j}$, respectively. The positive definite symmetric operator $A_{j}: M_{j} \rightarrow M_{j}$ is defined by

$$
\left(A_{j} V, W\right)_{j} \equiv A_{j}(V, W) \quad \forall V, W \in M_{j} .
$$

We also define operators $P_{j-1}: M_{j} \rightarrow M_{j-1}$ and $P_{j-1}^{0}: M_{j} \rightarrow M_{j-1}$ as:

$$
A_{j-1}\left(P_{j-1} V, W\right) \equiv A_{j}\left(V, I_{j} W\right)
$$

and

$$
\left(P_{j-1}^{0} V, W\right)_{j-1} \equiv\left(V, I_{j} W\right)_{j} \quad \forall V \in M_{j}, W \in M_{j-1} .
$$

It follows from (2.1a) that

$$
\left\|A_{j} V\right\|_{j}=\max _{0 \neq W \in M_{j}} \frac{\left|A_{j}(V, W)\right|}{\|W\|_{j}} .
$$

Remark 2.1. Suppose, for example, that $M_{j}$ is a conforming finite element space with mesh size $h_{j}, A_{j}(,) \equiv A($,$) corresponds to the bilinear form for$ the variational problem, and $(,)_{j}$ is equivalent to the $L^{2}$ inner product. In this case, the spaces $\left\{M_{j}\right\}$ are typically nested, i.e., $M_{j-1} \subseteq M_{j}$, and $I_{j}$ can be chosen to be the injection operator.

To define the smoothing process, we require a linear operator $R_{j}: M_{j} \rightarrow M_{j}$ for $j=1, \ldots, J$. We assume that $R_{j}$ is symmetric in the $(,)_{j}$ inner product and define

$$
K_{j} \equiv I-R_{j} A_{j},
$$

where $I$ denotes the identity operator. We next define a multigrid operator $B_{j}: M_{j} \rightarrow M_{j}$ by induction for each $j$. For each $g$ in $M_{j}, B_{j} g$ is to approximate $U=A_{j}^{-1} g$. 
Definition 2.1. Set $B_{0}=A_{0}^{-1}$. If $j>0$, assume that $B_{j-1}$ has been defined and define $B_{j} g$ for each $g$ in $M_{j}$ as follows (with prescribed integers $m_{j}>0$ and $\left.n_{j} \geq 0\right)$ :

(i) Set $U^{0}=0$.

(ii) Define $U^{l}$ for $l=1, \ldots, m_{j}$ by

$$
U^{l} \equiv U^{l-1}+R_{j}\left(g-A_{j} U^{l-1}\right) .
$$

(iii) Define $U^{m_{j}+1} \equiv U^{m_{j}}+I_{j} q$, where $q$ is defined by

$$
q \equiv B_{j-1} P_{j-1}^{0}\left(g-A_{j} U^{m_{j}}\right) \text {. }
$$

(iv) Set $B_{j} g \equiv U^{m_{j}+n_{j}+1}$, where $U^{l}$ is defined for $l=m_{j}+2, \ldots, m_{j}+$ $n_{j}+1$ by $(2.3)$.

This algorithm is referred to as a multigrid $\mathscr{V}$ cycle. $\mathscr{W}$-cycle algorithms can be defined analogously (see, e.g., [2, 3] or [9]). Since the initial iterate is zero, $B_{j}$ defines a linear operator acting in $M_{j} . I_{j}$ is referred to as a prolongation operator and its adjoint $P_{j-1}$ defined by $(2.1 \mathrm{~b})$ is a restriction operator.

Note that there are $m_{j}$ "presmoothings" in step (ii) and $n_{j}$ "postsmoothings" in step (iv). We consider the following two operators $B_{J}^{S}$ and $B_{J}^{N}$ :

$$
B_{J} \equiv B_{J}^{S} \quad \text { if } n_{j}=m_{j}, j=1, \ldots, J,
$$

and

$$
B_{J} \equiv B_{J}^{N} \quad \text { if } n_{j}=0, j=1, \ldots, J .
$$

It can be seen (e.g., using an induction argument and (2.7S) below) that $B_{J}^{S}$ defines a symmetric operator with respect to $A_{J}($,$) .$

For the usual $\mathscr{V}$-cycle algorithm, it is assumed that

$$
m_{j}=m \geq 1, \quad j=1, \ldots, J .
$$

We refer to this algorithm simply as the $\mathscr{V}$ cycle. Convergence estimates can sometimes be improved when the number of smoothings $m_{j}$ is increased on coarser grids. Such an algorithm, referred to as a variable $\mathscr{V}$ cycle, was defined in [9] by the following condition:

$$
\beta_{0} m_{j} \leq m_{j-1} \leq \beta_{1} m_{j}, \quad j=2, \ldots, J,
$$

where $\beta_{0}$ and $\beta_{1}$ are greater than one and independent of $j$. i.e.,

It is easily seen, using (2.2) and (2.3), that $K_{j}$ is an iteration matrix for $R_{j}$,

$$
U-U^{l}=K_{j}\left(U-U^{l-1}\right) \text { for } l=1, \ldots, m_{j} .
$$

The following important recurrence relations follow readily, using the preceding equality [9]:

$$
I-B_{j}^{N} A_{j}=\left[\left(I-I_{j} P_{j-1}\right)+I_{j}\left(I-B_{j-1}^{N} A_{j-1}\right) P_{j-1}\right] K_{j}^{m_{j}}
$$


and

$$
I-B_{j}^{S} A_{j}=K_{j}^{m_{j}}\left[\left(I-I_{j} P_{j-1}\right)+I_{j}\left(I-B_{j-1}^{S} A_{j-1}\right) P_{j-1}\right] K_{j}^{m_{j}} .
$$

In addition to equalities $(2.7 \mathrm{~N}, \mathrm{~S})$, the following assumption on the smoothing operator $R_{j}$ for each $j$ plays a key role in the analysis:

(A.1) The spectrum of the operator $K_{j}$ (with respect to the $A_{j}$ inner product) is contained in $[0,1)$. Furthermore, there is a positive constant $\widetilde{C}$, independent of $j$, such that

where $\lambda_{j}$ is the largest eigenvalue of $A_{j}$.

$$
\lambda_{j}^{-1}\|U\|_{j}^{2} \leq \widetilde{C}\left(R_{j} U, U\right)_{j} \quad \forall U \in M_{j},
$$

It was shown in $[3,9]$ and other references cited above that commonly used relaxation schemes (including Jacobi iteration) satisfy (A.1). It follows from the spectral properties of $K_{j}$ that

$$
\left\|K_{j} U\right\|_{A_{j}} \leq\|U\|_{A_{j}} \quad \forall U \in M_{j}, j=1, \ldots, J .
$$

The following lemma is crucial to the multigrid analysis.

Lemma 2.1. Suppose that (A.1) holds. Then

$$
\lambda_{j}^{-1}\left\|A_{j} K_{j}^{m_{j}} U\right\|_{j}^{2} \leq \widetilde{C}\left(2 m_{j}\right)^{-1} A_{j}\left(\left(I-K_{j}^{2 m_{j}}\right) U, U\right) \leq \widetilde{C}\left(2 m_{j}\right)^{-1}\|U\|_{A_{j}}^{2},
$$

where $m_{j} \geq 1$ and $\widetilde{C}$ is the constant in (A.1).

For a proof of the first estimate in (2.9), we refer to [9] (see (3.15) and (3.16) in the proof of Theorem 1 there). The last estimate in (2.9) then follows immediately, since $K_{j}$ is a nonnegative operator by (A.1).

2.2. Convergence estimates. In Theorem 2.1 below, we state convergence estimates for the multigrid operators $B_{J}^{N}$ and $B_{J}^{S}$. For $B_{J}^{N}$, the convergence estimate is expressed in the form

$$
\left\|\left(I-B_{J}^{N} A_{J}\right) U\right\|_{A_{J}}^{2} \leq \delta\|U\|_{A_{J}}^{2} \quad \forall U \in M_{J}
$$

with suitable values of $\delta \in[0,1)$. For $B_{J}^{S}$, the convergence estimate is expressed in the form

$$
0 \leq A_{J}\left(\left(I-B_{J}^{S} A_{J}\right) U, U\right) \leq \delta\|U\|_{A_{J}}^{2} \quad \forall U \in M_{J} .
$$

Estimates $(2.10 \mathrm{~S}, \mathrm{~N})$ can be proved using the following additional assumptions:

$$
A_{j}\left(I_{j} U, I_{j} V\right)=A_{j-1}(U, V) \quad \forall U, V \in M_{j-1}, j=1, \ldots, J
$$

and

$$
\text { (A.3) } \quad A_{j}\left(\left(I-I_{j} P_{j-1}\right) U, U\right) \leq C_{1} \lambda_{j}^{-1}\left\|A_{j} U\right\|_{j}^{2} \quad \forall U \in M_{j}, j=1, \ldots, J \text {, }
$$

where $C_{1}$ is independent of $j$. Condition (A.2) (or more generally, (A.2S) below) implies that the form $A_{j-1}($,$) on the space M_{j-1}$ can be related to 
that on $M_{j}$. It follows from (A.2S) that the operator norm of $I_{j}$ and its adjoint $P_{j-1}$ is bounded by one. This implies that the left side of (A.3) is nonnegative. Furthermore, the first inequality in $(2.10 \mathrm{~S})$ follows readily from (A.2S), (2.7S), and induction.

Theorem 2.1. Suppose that $B_{J}$ is the $\mathscr{V}$-cycle or variable $\mathscr{V}$-cycle algorithm defined by Definition 2.1, $m \equiv m_{J} \geq 1$, and conditions (A.1)-(A.3) hold. If (2.5S) holds, then (2.10S) holds with

$$
\delta=C_{0} /\left(C_{0}+m\right),
$$

where $C_{0}=\widetilde{C} C_{1} / 2$ is independent of $J$ and $m$. If $(2.5 \mathrm{~N})$ holds, then $(2.10 \mathrm{~N})$ holds with $\delta$ given by (2.11).

This result was first proved in [6] for the $\mathscr{V}$ cycle. The variable $\mathscr{V}$ cycle was analyzed in [9].

Remark 2.2. When multigrid is applied to the finite element method as in Remark 2.1, condition (A.2) follows immediately (assuming $A_{j}(,) \equiv A($,$) is$ calculated exactly on each grid level). This is not true in general when $A_{j}($,$) is$ approximated using numerical quadrature. If we assume a quasi-uniform mesh for each $j$, with mesh size $h_{j}<h_{j-1}$, then it follows from standard inverse inequalities [13] that

$$
C^{-1} h_{j}^{-2} \leq \lambda_{j} \leq C h_{j}^{-2}
$$

In this case, (A.3) follows using standard finite element $H^{1}$ error estimates, assuming $H^{2}$ regularity for solutions of the given boundary value problem and each $A_{j}(,) \equiv A($,$) is calculated exactly (see, e.g., [2, 3] or [9]). Hence,$ Theorem 2.1 is applicable in this case. $\mathscr{V}$-cycle convergence estimates under weaker regularity assumptions were proved in $[9,12]$.

Note. We shall use the same letter $C$ to denote different constants, independent of $j$, when there is no danger of confusion.

An analogue of Theorem 2.1 was proved in [16] for the symmetric operator $B_{J}^{S}$ with (A.2) replaced by the following more general condition:

$$
\text { (A.2S) } \quad A_{j}\left(I_{j} U, I_{j} U\right) \leq A_{j-1}(U, U) \quad \forall U \in M_{j-1}, j=1, \ldots, J .
$$

In the next section, we consider a more general multigrid framework for which (A.2S) does not hold. Such a generalization is required for the application to numerical quadrature in $\S 4$ (as well as other important applications).

\section{EXTENSIONS OF THE THEORY}

In this section we state some multigrid results with weaker assumptions than (A.2S) and (A.3). These results will be applied to numerical quadrature in the next section. The results in $\S 3.1$ for the symmetric operator $B_{J}^{S}$ follow immediately from the results and techniques in [16]. In $\S 3.2$, we state some convergence results proved in the Appendix for both $B_{J}^{N}$ and $B_{J}^{S}$. 
3.1. Symmetric multigrid operators. We assume that (A.1) holds. As shown in [16], $B_{J}^{S} A_{J}$ is a positive definite symmetric operator with respect to $A_{J}($, ) for any $m_{J} \geq 1$. Hence $B_{J}^{S}$ can be used as a preconditioner for an iterative method such as the conjugate gradient method. Estimates of the convergence rate of the resulting preconditioned algorithm depend on estimates for the largest and smallest eigenvalues of $B_{J}^{S} A_{J}$ [18]. These eigenvalues, $\eta_{1}$ and $\eta_{0}$, satisfy the inequalities

$$
\eta_{0}\|U\|_{A_{J}}^{2} \leq A_{J}\left(B_{J}^{S} A_{J} U, U\right) \leq \eta_{1}\|U\|_{A_{J}}^{2} \quad \forall U \in M_{J}
$$

If (3.1) holds for some $\eta_{1}, \eta_{0}>0$, the condition number $\kappa$ satisfies $\kappa\left(B_{J}^{S} A_{J}\right)$ $=O\left(\eta_{1} / \eta_{0}\right)$ and the preconditioned conjugate gradient method converges in $O\left(\left(\eta_{1} / \eta_{0}\right)^{1 / 2}\right)$ iterations to a prescribed accuracy. The following generalization of (A.3) was assumed in [16] in order to estimate the positive constants, $\eta_{0}$ and $\eta_{1}$

(A.4) There is an $\alpha \in(0,1]$ such that $\forall U \in M_{j}, j=1, \ldots, J$, we have

$$
\left|A_{j}\left(\left(I-I_{j} P_{j-1}\right) U, U\right)\right| \leq C_{\alpha}\left(\lambda_{j}^{-1}\left\|A_{j} U\right\|_{j}^{2}\right)^{\alpha} A_{j}(U, U)^{1-\alpha} .
$$

The next two theorems were proved in [16] and show that $\kappa\left(B_{J}^{S} A_{J}\right)=O(1)$ for the variable $\mathscr{V}$ cycle and $O\left(1+J^{(1-\alpha) / \alpha}\right)$ for the $\mathscr{V}$ cycle as $J \rightarrow \infty$.

Theorem 3.1. Suppose that $B_{J}^{S}$ is the symmetric variable $\mathscr{V}$-cycle algorithm defined by Definition 2.1 and (2.6b). Assume that (A.1) and (A.4) hold. Then for any $m \equiv m_{J} \geq 1$, the constants $\eta_{0}$ and $\eta_{1}$ in (3.1) satisfy

$$
\eta_{0} \geq \frac{m^{\alpha}}{C_{0}+m^{\alpha}}
$$

and

$$
\eta_{1} \leq \frac{C_{0}+m^{\alpha}}{m^{\alpha}}
$$

where $C_{0}$ is independent of $J$ and $m$. Hence, $\kappa\left(B_{J}^{S} A_{J}\right)=O(1)$ as $J \rightarrow \infty$.

To prove an analogous result for the standard $\mathscr{V}$-cycle algorithm, an additional condition was assumed in [16] that can be stated as follows:

$$
A_{j}\left(\left(I-I_{j} P_{j-1}\right) U, U\right) \geq-E_{j}\left(U, \lambda_{j}\right) \equiv-C_{2} \lambda_{j}^{-\sigma}\|U\|_{A_{j}}^{2} \quad \forall U \in M_{j} .
$$

We shall also find the following condition useful:

$$
\begin{aligned}
A_{j}\left(\left(I-I_{j} P_{j-1}\right) U, U\right) & \geq-E_{j}\left(U, \lambda_{j}\right) \\
& \equiv-C_{2} \lambda_{j}^{-(\sigma+1 / 2)}\left\|A_{j} U\right\|_{j}\|U\|_{A_{j}} \quad \forall U \in M_{j} .
\end{aligned}
$$


We make the following assumption for the remainder of this section:

(A.5) There are positive constants, $\sigma$ and $C_{2}$, independent of $j=1, \ldots, J$, such that either (3.3a) or (3.3b) holds.

We now state an analogue of Theorem 3.1 for the $\mathscr{V}$ cycle. This result follows from Theorem 8 in [16] (since (3.3a) follows from (3.3b) by Lemma A.1 in the Appendix).

Theorem 3.2. Suppose that $B_{J}^{S}$ is the symmetric $\mathscr{V}$-cycle algorithm defined by Definition 2.1 and (2.6a). Assume that (A.1), (A.4), and (A.5) hold and $\lambda_{j}$ satisfies

$$
\lambda_{j}^{-1} \leq \gamma^{j}, \quad j=1, \ldots, J,
$$

for some $\gamma<1$ and independent of $j$. Then for any $m \equiv m_{J} \geq 1, \eta_{1}$ in (3.1) can be chosen independently of $J$, and $\eta_{0}$ satisfies

$$
\eta_{0} \geq \frac{m^{\alpha}}{C_{0} J^{(1-\alpha) / \alpha}+m^{\alpha}},
$$

where $C_{0}$ is independent of $J$ and $m$. Hence,

$$
\kappa\left(B_{J}^{S} A_{J}\right)=O\left(1+J^{(1-\alpha) / \alpha}\right) \text { as } J \rightarrow \infty .
$$

Since $A_{j}$ is positive definite symmetric, it follows that

$$
\|U\|_{A_{j}}^{2} \leq C_{3}\left\|A_{j} U\right\|_{j}^{2} \quad \forall U \in M_{j}
$$

with $C_{3}>0$. We assume that $C_{3}$ is independent of $j$, or equivalently, that the smallest eigenvalue of $A_{j}$ is bounded away from zero, uniformly for all $j$. This is the case in typical applications (e.g., \|\|$_{j}$ and \|\|$_{A_{j}}$ are respectively equivalent to \|\|$_{L^{2}}$ and \|\|$_{H^{1}}$, uniformly in $j$ for the application in $\S 4$ and elsewhere).

We next state a generalization of Theorem 2.1 with $B_{J}=B_{J}^{S}$. The proof of this result is a simple consequence of Theorems 3.1 and 3.2 and is included in the Appendix for the sake of completeness.

Theorem 3.3. Suppose that (A.1), (A.4), (A.5), and (3.4) hold. Then

$$
A_{J}\left(\left(I-B_{J}^{S} A_{J}\right) U, U\right) \leq \delta_{J}^{(2)}\|U\|_{A_{J}}^{2} \quad \forall U \in M_{J},
$$

where

$$
\delta_{J}^{(2)}=\frac{C_{0} J^{(1-\alpha) / \alpha}}{C_{0} J^{(1-\alpha) / \alpha}+m_{J}^{\alpha}} \quad \text { for the } \mathscr{V} \text { cycle }
$$

and

$$
\delta_{J}^{(2)}=\frac{C_{0}}{C_{0}+m_{J}^{\alpha}} \text { for the variable } \mathscr{V} \text { cycle. }
$$

Furthermore,

$$
A_{J}\left(\left(I-B_{J}^{S} A_{J}\right) U, U\right) \geq\left(1-\eta_{1}\right)\|U\|_{A_{J}}^{2}
$$


with $\eta_{1}$ defined as follows. If (3.3a) holds and $\sigma_{0} \leq 1$ satisfies $0<\sigma_{0}<\sigma$, then

$$
\eta_{1}=\prod_{j=1}^{J}\left(1+C C_{2} m_{j}^{-\sigma_{0}} \lambda_{j}^{-\left(\sigma-\sigma_{0}\right)}\right) \leq 1+C C_{2} m_{J}^{-\sigma_{0}} .
$$

If (3.3b) holds and $\sigma_{0} \leq 1 / 2$ satisfies $0<\sigma_{0}<\sigma$, then

$$
\eta_{1}=\prod_{j=1}^{J}\left(1+C C_{2} m_{j}^{-\left(1 / 2+\sigma_{0}\right)} \lambda_{j}^{-\left(\sigma-\sigma_{0}\right)}\right) \leq 1+C C_{2} m_{J}^{-\left(\sigma_{0}+1 / 2\right)} .
$$

Finally,

$$
\left|A_{J}\left(\left(I-B_{J}^{S} A_{J}\right) U, U\right)\right| \leq \delta_{J}\|U\|_{A_{J}}^{2},
$$

where

$$
\delta_{J}=\max \left(\delta_{J}^{(2)}, \eta_{1}-1\right)
$$

and $\delta_{J}<1$ for $m_{J}$ sufficiently large.

Remark 3.1. If $C_{2}=0$ in (3.3a) or (3.3b), then (A.5) reduces to (A.2S). As noted earlier, (2.12) holds in many applications. Hence, condition (A.5) is a perturbation of (A.2S) up to some power of the grid size $h_{j}$. It now follows that the additional error due to this perturbation at each grid level is bounded by $O\left(h_{j}^{\sigma_{1}} m_{j}^{-\sigma_{2}}\right)$ (see the second term in the parentheses in (3.9b) or (3.9c)), where the positive exponents, $\sigma_{1}$ and $\sigma_{2}$, can be calculated from $E_{j}$ in (3.3a) or (3.3b). Analogous assertions hold for Theorems $3.4 \mathrm{~N}$ and $3.4 \mathrm{~S}$ below. We shall see in $\S 4$ how $E_{j}$ depends on the accuracy of the numerical quadrature scheme on the $j$ th grid level.

3.2. Additional results. In Theorem $3.4 \mathrm{~N}$ below, we state a generalization of Theorem 2.1 for the nonsymmetric operator $B_{J}^{N}$ corresponding to the $\mathscr{V}$ or variable $\mathscr{V}$ cycle. To this end, we replace (A.4) by the following perturbation of (A.3):

(A.6) There is a positive constant, $C_{1}$, independent of $j=1, \ldots, J$, such that

$$
A_{j}\left(\left(I-I_{j} P_{j-1}\right) U, U\right) \leq C_{1} \lambda_{j}^{-1}\left\|A_{j} U\right\|_{j}^{2}+E_{j}\left(U, \lambda_{j}\right)
$$

with $E_{j}\left(U, \lambda_{j}\right)$ defined by either (3.3a) or (3.3b).

We also replace (A.5) by the following perturbation of condition (A.2):

(A.7) There are positive constants, $\sigma$ and $C_{2}$, independent of $j=1, \ldots, J$, such that either

$$
\left\|\left(I-P_{j-1} I_{j}\right) U\right\|_{A_{j-1}} \leq F_{j}\left(U, \lambda_{j}\right) \equiv C_{2} \lambda_{j}^{-\sigma}\|U\|_{A_{j-1}}
$$

or

$$
\left\|\left(I-P_{j-1} I_{j} U\right)\right\|_{A_{j-1}} \leq F_{j}\left(U, \lambda_{j}\right) \equiv C_{2} \lambda_{j}^{-(\sigma+1 / 2)}\left\|A_{j-1} U\right\|_{j-1} .
$$


The following theorem is proved in the Appendix, assuming $C^{-1} \lambda_{j-1}^{-1} \leq$ $\lambda_{j}^{-1} \leq C \lambda_{j-1}^{-1}$ for each $j$. This estimate typically holds in applications (see, e.g., (2.12)). Furthermore, we may assume that $\sigma$ is the same constant in (A.5)-(A.7).

Theorem 3.4N. Assume that (A.1), (A.6), (A.7), and (3.4) hold. Also assume that $0<\sigma_{0}<\sigma$, where $\sigma$ is the constant in (A.6) and (A.7) and $\sigma_{0} \leq 1$. Then

$$
\left\|\left(I-B_{J}^{N} A_{J}\right) U\right\|_{A_{J}}^{2} \leq \tilde{\delta}\|U\|_{A_{J}}^{2} \quad \forall U \in M_{J}
$$

with

$$
\tilde{\delta}=\delta+C C_{2} \sum_{j=1}^{J} \lambda_{j}^{-\left(\sigma-\sigma_{0}\right)} m_{j}^{-\sigma_{0}} \leq C m_{J}^{-\sigma_{0}} .
$$

Here, $\delta$ is expressed in terms of the constants $\widetilde{C}$ and $C_{1}$ in (A.1) and (A.6) by

$$
\delta=C_{0} /\left(C_{0}+m_{J}\right) \text {, }
$$

where

$$
C_{0}=\widetilde{C} C_{1} / 2 .
$$

Hence, for $m_{J}$ sufficiently large (and independent of $J$ ), we have

$$
\tilde{\delta}<1 .
$$

Thus, $I-B_{J}^{N} A_{J}$ is a contraction operator for $m_{J}$ sufficiently large with contraction number independent of $J$. Note that when $C_{2}$ is zero in (3.3) and (3.12), Theorem $3.4 \mathrm{~N}$ is the same as Theorem 2.1 with $B_{J}=B_{J}^{N}$. Finally, we state an analogue of Theorem $3.4 \mathrm{~N}$ for $B_{J}^{S}$ with (A.7) replaced by the more general condition (A.5). The proof of this result is similar to that of Theorem $3.4 \mathrm{~N}$ and is also given in the Appendix.

Theorem 3.4S. Assume that the hypotheses of Theorem 3.4 hold with (A.7) replaced by (A.5). Then

$$
A_{J}\left(\left(I-B_{J}^{S} A_{J}\right) U, U\right) \leq \delta^{(2)}\|U\|_{A_{J}}^{2} \quad \forall U \in M_{J},
$$

where

$$
\delta^{(2)}=\delta+C C_{2} \sum_{j=1}^{J} \lambda_{j}^{-\left(\sigma-\sigma_{0}\right)} m_{j}^{-\sigma_{0}} .
$$

Here, $\delta$ is again given by $(3.14 \mathrm{a}, \mathrm{b})$. Furthermore,

$$
\left|A_{J}\left(\left(I-B_{J}^{S} A_{J}\right) U, U\right)\right| \leq \tilde{\delta}\|U\|_{A_{J}}^{2} \quad \forall U \in M_{J},
$$

where

$$
\tilde{\delta}=\max \left(\delta^{(2)}, \eta_{1}-1\right) \leq C m_{J}^{-\sigma}
$$


with $\eta_{1}$ given by (3.9b) or (3.9c). Hence, for $m_{J}$ sufficiently large (and independent of $J$ ), we have

$$
\tilde{\delta}<1
$$

\section{NUMERICAL INTEGRATION}

In this section, we use the results in $\S 3$ to establish multigrid convergence estimates for the system of linear equations arising when a numerical quadrature scheme is used to compute the stiffness matrix for the finite element discretization of the following two-dimensional elliptic boundary value problem:

$$
\begin{aligned}
-\left\{\frac{\partial}{\partial x_{1}}\left(a_{11} \frac{\partial u}{\partial x_{1}}\right)+\frac{\partial}{\partial x_{2}}\left(a_{12} \frac{\partial u}{\partial x_{1}}\right)\right. \\
\left.+\frac{\partial}{\partial x_{1}}\left(a_{12} \frac{\partial u}{\partial x_{2}}\right)+\frac{\partial}{\partial x_{2}}\left(a_{22} \frac{\partial u}{\partial x_{2}}\right)\right\}+a u=f \text { in } \Omega,
\end{aligned}
$$

where $\Omega$ is a convex polygon, $f \in L^{2}(\Omega)$, the coefficients are real-valued, $a(x) \geq 0$ in $\bar{\Omega} \equiv \Omega \cup \partial \Omega$, and $x=\left(x_{1}, x_{2}\right) \in \bar{\Omega}$. We assume that the operator is uniformly elliptic, i.e., there is a positive constant $C$ such that

$$
C^{-1}\left(\xi_{1}^{2}+\xi_{2}^{2}\right) \leq a_{11} \xi_{1}^{2}+2 a_{12} \xi_{1} \xi_{2}+a_{22} \xi_{2}^{2} \leq C\left(\xi_{1}^{2}+\xi_{2}^{2}\right)
$$

for each $x \in \bar{\Omega}$ and real $\xi_{1}, \xi_{2}$.

In $\S 4.1$, we discuss the finite element method with numerical quadrature and present some preliminary results. In particular, we show in Theorem 4.1 that conditions (A.4)-(A.7) hold. In $\S 4.2$, we prove our multigrid results.

Note. We consider Dirichlet boundary conditions and triangular partitions. However, analogous results may be proved in the same way for Neumann and other boundary conditions as well as rectangular partitions and more general domains, assuming $H^{2}$ regularity (see (4.2) below).

4.1. Quadrature estimates. We begin by introducing some notation. Let $H^{s}(\Omega)$ denote the Sobolev space consisting of square-integrable functions with squareintegrable derivatives up to order $s \geq 0$, and let \|\|$_{H^{s}} \equiv\|\|_{H^{s}(\Omega)}$ denote the corresponding Sobolev norm [19]. The inner product and norm in $L^{2}(\Omega)=$ $H^{0}(\Omega)$ are denoted by $(, \quad)$ and \|\|$_{L^{2}} \equiv\|\|_{L^{2}(\Omega)}$, respectively. Since $\Omega$ is convex, we have for sufficiently smooth coefficients in (4.1a),

$$
\|u\|_{H^{2}} \leq C\|f\|_{L^{2}} .
$$

We consider the weak or variational formulation of $(4.1 \mathrm{a}, \mathrm{b})$. The energy subspace $H^{E} \subset H^{1}(\Omega)$ consists of functions with vanishing trace on $\partial \Omega$. The solution of (4.1) satisfies

$$
\widehat{A}(u, v)=(f, v) \quad \forall v \in H^{E},
$$


where for each $v, w \in H^{E}$,

$$
\begin{aligned}
\widehat{A}(v, w) \equiv \int_{\Omega}\left(a_{11} \frac{\partial v}{\partial x_{1}} \frac{\partial w}{\partial x_{1}}+a_{12}\left(\frac{\partial v}{\partial x_{1}} \frac{\partial w}{\partial x_{2}}\right.\right. & \left.+\frac{\partial v}{\partial x_{2}} \frac{\partial w}{\partial x_{1}}\right) \\
& \left.+a_{22} \frac{\partial v}{\partial x_{2}} \frac{\partial w}{\partial x_{2}}+a v w\right) d x .
\end{aligned}
$$

We next define the finite element spaces. Let $P^{k}(S)$ denote the set of polynomials of degree at most $k$ defined on the set $S$, where $k \geq 1$. Suppose that $\Omega=\bigcup_{\tau_{J}^{i} \in T_{J}} \tau_{J}^{i}$ gives a quasi-uniform triangulation $T_{J}$ of $\Omega$ into triangles $\tau_{J}^{i}$ with $\operatorname{diam}\left(\tau_{J}^{i}\right)=O\left(h_{J}\right)$. The finite element space $M_{J} \subset H^{E}$ consists of continuous functions $V_{J}$ vanishing on $\partial \Omega$, such that each $\left.V_{J}^{i} \equiv V_{J}\right|_{\tau_{J}^{i}} \in P^{k}\left(\tau_{J}^{i}\right)$.

We assume that there is a nested sequence of spaces $\left\{M_{j}\right\}$ such that each $M_{j}$ is defined in the same way as $M_{J}$. To define these spaces, suppose there is a sequence of quasi-uniform triangulations $T_{j}$ of $\Omega$, where the quasi-uniformity constants are independent of $j$. The triangulations are nested in the sense that any $\tau_{j-1}^{i}$ in $T_{j-1}$ can be written as a union of elements $\tau_{j}^{i}$ in $T_{j}$. We assume that $\operatorname{diam}\left(\tau_{j}^{i}\right)=O\left(h_{j}\right)$, where

$$
h_{j-1}=2 h_{j}, \quad j=1, \ldots, J .
$$

As in [13], we assume that $M_{j}$ is obtained by means of an invertible affine mapping of each $\tau_{j}^{i} \in T_{j}$ onto a reference triangle $\tau$ with $\operatorname{diam}(\tau)=1$. Hence, each $V_{j}^{i}$ is mapped into a polynomial $p \in P^{k}(\tau)$. We say that the finite element space $M_{j}$ has degree $k$.

We approximate the exact integrals on the right side of $(4.3 \mathrm{~b})$ by defining a numerical quadrature scheme $Q_{j}^{i}$ over each element $\tau_{j}^{i} \in T_{j}$. To be specific, first consider the reference triangle $\tau$ and approximate $\int_{\tau} \hat{\phi}(\hat{x}) d \hat{x}$ as follows:

$$
\int_{\tau} \hat{\phi}(\hat{x}) d \hat{x} \approx \sum_{l=1}^{L} \omega_{l} \hat{\phi}\left(b_{l}\right)
$$

where the $\omega_{l}$ are positive weights and the $b_{l} \in \tau$ are quadrature points. We then define the quadrature rule on each $\tau_{j}^{i}$ by

$$
\int_{\tau_{j}^{i}} \phi(x) d x \approx \sum_{l=1}^{L} \omega_{j, l}^{i} \phi\left(b_{j, l}^{i}\right) \equiv Q_{j}^{i}[\phi],
$$

where $\phi(x)=\hat{\phi}(\hat{x})$ and the weights $\omega_{j, l}^{i}$ and quadrature points $b_{j, l}^{i}$ are defined in terms of the $\omega_{l}$ and $b_{l}$ by means of the affine mapping from $\tau_{j}^{i}$ onto $\tau$ that takes each $x$ in $\tau_{j}^{i}$ into $\hat{x}$ in $\tau$. We refer to $[13,14]$ for detailed descriptions of numerical integration in connection with the finite element method.

We define the quadrature error functionals $E_{j}^{i}$ by

$$
E_{j}^{i}[\phi] \equiv \int_{\tau_{j}^{i}} \phi(x) d x-Q_{j}^{i}[\phi] .
$$


Our main results require that the quadrature is exact for polynomials of sufficiently large degree. This was also the case for the finite element analysis (see [13] for energy and $L^{2}$ error estimates and [15] for $L^{\infty}$ estimates). To be precise, we assume that for each $\tau_{j}^{i} \in T_{j}$,

$$
E_{j}^{i}[\phi]=0 \quad \forall \phi \in P^{2 k-2+L}\left(\tau_{j}^{i}\right)
$$

for some integer $L \geq 0$. See $[13, \S 4.2]$ and references cited there for specific examples of schemes satisfying (4.5).

Note. Condition (4.5) is known as the patch test. It was proved in [13] that when $L=0$ in (4.5), optimal energy (i.e., $H^{1}$ ) error estimates hold with sufficient regularity assumptions. Hence, e.g., when $M_{j}$ consists of continuous piecewise linear functions, it suffices for the quadrature scheme to be exact for constants.

To approximate the right side of (4.3b) using the quadrature scheme, we define the bilinear form $A_{j}($,$) acting on M_{j} \times M_{j}$ as follows, for each $j=$ $0, \ldots, J$ and $U, V \in M_{j}$ :

$$
\begin{aligned}
A_{j}(U, V) \equiv \sum_{\tau_{j}^{i} \in T_{j}} Q_{j}^{i}\left[a_{11} \frac{\partial U}{\partial x_{1}} \frac{\partial V}{\partial x_{1}}+a_{12}\left(\frac{\partial U}{\partial x_{1}}\right.\right. & \left.\frac{\partial V}{\partial x_{2}}+\frac{\partial U}{\partial x_{2}} \frac{\partial V}{\partial x_{1}}\right) \\
& \left.+a_{22} \frac{\partial U}{\partial x_{2}} \frac{\partial V}{\partial x_{2}}+a U V\right] .
\end{aligned}
$$

We shall see from Lemma 4.1 below that $A_{j}($,$) defines a positive definite$ symmetric bilinear form on $M_{j} \times M_{j}$. Define the operator $A_{j}: M_{j} \rightarrow M_{j}$ by (2.1a) with $(,)_{j} \equiv($,$) . Denote the largest eigenvalue of A_{j}$ by $\lambda_{j}$. The approximate solution $U \in M_{J}$ of (4.1) satisfies

$$
\left(A_{J} U, V\right) \equiv A_{J}(U, V)=(f, V) \quad \forall V \in M_{J} .
$$

We wish to solve (4.7) using the multigrid algorithm in $\S 2$. Let $R_{j}: M_{j} \rightarrow M_{j}$ denote any smoothing operator satisfying (A.1) with $(,)_{j} \equiv() .,\left(R_{j}\right.$ may be defined in the same way as for the finite element method without numerical integration.) Let $I_{j}$ denote the injection operator from $M_{j-1}$ into $M_{j}$ and define $P_{j-1}$ by

$$
A_{j-1}\left(P_{j-1} U, V\right)=A_{j}\left(U, I_{j} V\right) \quad \forall U \in M_{j}, V \in M_{j-1} .
$$

The multigrid operator $B_{j}=B_{j}^{S}$ or $B_{j}^{N}$ is now defined by Definition 2.1. Condition (A.2S) does not hold in general for this case.

Remark 4.1. To avoid the inversion of $L^{2}$ Gram matrices, we may replace (, ) by other more convenient inner products that are equivalent to $($,$) , i.e.,$

$$
C^{-1}\|U\|_{j}^{2} \leq\|U\|_{L^{2}}^{2} \leq C\|U\|_{j}^{2} \quad \forall U \in M_{j} .
$$

Specific forms $(,)_{j}$ satisfying this condition and smoothing operators symmetric in $(,)_{j}$ and satisfying (A.1) can be obtained, e.g., as in $[2,3,7,9]$. 
Now define $A_{j}^{\prime}$ by (2.1a) and let $\lambda_{j}^{\prime}$ denote the largest eigenvalue of $A_{j}^{\prime}$. Since $($,$) is equivalent to (,)_{j}$ and the same form $A_{j}($,$) is used to define both$ $A_{j}$ and $A_{j}^{\prime}$, it follows by applying $(2.1 \mathrm{~d})$ to both $A_{j}$ and $A_{j}^{\prime}$ that

$$
C^{-1}\left\|A_{j}^{\prime} V\right\|_{j} \leq\left\|A_{j} V\right\|_{L^{2}} \leq C\left\|A_{j}^{\prime} V\right\|_{j} \quad \forall V \in M_{j} .
$$

Similarly, it follows by applying the first equation in (4.13) below to both $\lambda_{j}$ and $\lambda_{j}^{\prime}$ that

$$
C^{-1} \lambda_{j}^{\prime} \leq \lambda_{j} \leq C \lambda_{j}^{\prime}
$$

With $I_{j}$ again denoting the injection operator, it now follows readily that (A.4)(A.7) hold with respect to $(,)_{j}$ if and only if they hold with respect to $($,$) .$ Hence it suffices to prove our results for $($,$) .$

We also require the orthogonal projection operator $\widehat{P}_{j-1}: M_{j} \rightarrow M_{j-1}$ corresponding to the unperturbed bilinear form $\widehat{A}($,$) in (4.3 \mathrm{~b})$ :

$$
\widehat{A}\left(\widehat{P}_{j-1} U, V\right) \equiv \widehat{A}\left(U, I_{j} V\right) \quad \forall U \in M_{j}, V \in M_{j-1} .
$$

Condition (A.2) clearly holds with respect to $\widehat{A}($,$) . Let \hat{\lambda}_{j}$ denote the largest eigenvalue of the operator $\widehat{A}_{j}$ corresponding to $\widehat{A}($,$) . As noted in Remark$ 2.2, (A.3) holds with $A_{j}, \lambda_{j}$, etc. replaced by $\widehat{A}_{j}, \hat{\lambda}_{j}$, etc. The following result shows that $\widehat{A}($,$) and A_{j}($,$) are equivalent bilinear forms.$

Lemma 4.1. Suppose that $M_{j}$ has degree $k \geq 1$ and (4.5) holds with $L=0$. Then

$$
C^{-1} \widehat{A}(U, U) \leq A_{j}(U, U) \leq C \widehat{A}(U, U) \quad \forall U \in M_{j} .
$$

Lemma 4.1 follows from [13, Theorem 4.1.2]. In view of (4.1c), (4.3b), and (4.10), there are positive constants, $C$ and $C^{\prime}$, independent of $j$, such that

$$
\begin{aligned}
C^{\prime-1}\|U\|_{H^{1}(\Omega)} & \leq C^{-1}\|U\|_{\widehat{A}} \\
& \leq\|U\|_{A_{j}} \leq C\|U\|_{\widehat{A}} \leq C^{\prime}\|U\|_{H^{1}(\Omega)} \quad \forall U \in M_{j} .
\end{aligned}
$$

Lemma 4.2. Suppose that the hypotheses of Lemma 4.1 hold. Then there are positive constants, $C$ and $C^{\prime}$, independent of $j$, such that

$$
C^{\prime-1} h_{j}^{-2} \leq C^{-1} \hat{\lambda}_{j} \leq \lambda_{j} \leq C \hat{\lambda}_{j} \leq C^{\prime} h_{j}^{-2} \text {. }
$$

Proof. Since $A_{j}$ and $\widehat{A}$ are positive definite symmetric operators, we have

$$
\lambda_{j}=\max _{0 \neq V \in M_{j}} \frac{A_{j}(V, V)}{(V, V)} \quad \text { and } \quad \hat{\lambda}_{j}=\max _{0 \neq V \in M_{j}} \frac{\widehat{A}(V, V)}{(V, V)} .
$$

(4.12) now follows from (4.10), (4.13), and the quasi-uniformity of the mesh.

Let $W_{\infty}^{s}(S)$ denote the Sobolev space of real-valued functions $v$ defined on $S$ such that

$$
\|v\|_{W_{\infty}^{s}(S)} \equiv \max _{|\beta| \leq s}\left\|D^{\beta} v\right\|_{L^{\infty}(S)}<\infty
$$


where $s$ is a nonnegative integer and $D^{\beta} v$ denotes the weak derivative of $v$ [19].

Lemma 4.3. Suppose that $\tau_{j}^{i} \in T_{j}, k \geq 1, b \in W_{\infty}^{k+L}\left(\tau_{j}^{i}\right)$, and (4.5) holds for some $L \geq 0$. Then there is a positive constant $C$, independent of $j=0, \ldots, J$ and $\tau_{j}^{i} \in T_{j}$, such that for each $p^{\prime}, p \in P^{k}\left(\tau_{j}^{i}\right)$, we have

$$
\begin{aligned}
& \sum_{l_{1}, l_{2}=1}^{2}\left|E_{j}^{i}\left[b \frac{\partial p^{\prime}}{\partial x_{l_{1}}} \frac{\partial p}{\partial x_{l_{2}}}\right]\right|+\left|E_{j}^{i}\left[b p^{\prime} p\right]\right| \\
& \quad \leq C h_{j}^{k+L}\|b\|_{W_{\infty}^{k+L}\left(\tau_{j}^{i}\right)}\|p\|_{H^{1}\left(\tau_{j}^{i}\right)}\left\|p^{\prime}\right\|_{H^{k}\left(\tau_{j}^{i}\right)} .
\end{aligned}
$$

The proof of Lemma 4.3 is essentially the same as that of Theorem 4.1.4 in [13], and hence will be omitted. (Note that $L=0$ for the result cited in [13], since this is sufficient for the finite element error estimates proved there. However, the same argument goes through for $L>0$.)

In order to prove Theorem 4.1 below, we first prove the following key result.

Lemma 4.4. Suppose that $a_{11}(x), a_{12}(x), a_{22}(x), a(x) \in W_{\infty}^{k+L}(\Omega)$. Also assume that $M_{j}$ has degree $k \geq 1$ and (4.5) holds for some $L \geq 0$. Then for each $U, V \in M_{j}$,

$$
\left|\widehat{A}(U, V)-A_{j}(U, V)\right| \leq C h_{j}^{L+1}\|V\|_{H^{1}(\Omega)}\|U\|_{H^{1}(\Omega)} \quad \text { if } k=1
$$

and

$$
\left|\widehat{A}(U, V)-A_{j}(U, V)\right| \leq C h_{j}^{L+2}\|V\|_{H^{1}(\Omega)}\left\|\widehat{A_{j}} U\right\|_{L^{2}(\Omega)} \quad \text { if } k>1 .
$$

Alternatively, (4.15b) can be replaced by

$$
\left|\widehat{A}(U, V)-A_{j}(U, V)\right| \leq C h_{j}^{L+2}\|V\|_{H^{1}(\Omega)}\left\|A_{j} U\right\|_{L^{2}(\Omega)} \quad \text { if } k>1 .
$$

Proof. Suppose that $U, V \in M_{j}$ and note that $\left.U\right|_{\tau_{j}^{i}},\left.V\right|_{\tau_{j}^{i}} \in P^{k}\left(\tau_{j}^{i}\right)$. Applying (4.3b), (4.6), Lemma 4.3, and the Schwarz inequality, we obtain

$$
\left|\widehat{A}(U, V)-A_{j}(U, V)\right| \leq C h_{j}^{k+L}\|V\|_{H^{1}(\Omega)} \cdot\left(\sum_{\tau_{j}^{i} \in T_{j}}\|U\|_{H^{k}\left(\tau_{j}^{i}\right)}^{2}\right)^{1 / 2},
$$

where $C$ depends on the $W_{\infty}^{k+L}(\Omega)$ norm of the coefficients, but is independent of $j=0, \ldots, J$. (4.15a) now follows immediately from (4.16), since $M_{j} \subset$ $H^{E}$. Hence, we are left with proving $(4.15 \mathrm{~b}, \mathrm{c})$ (i.e., $\left.k>1\right)$.

To prove $(4.15 \mathrm{~b})$, let $u_{j}$ denote the solution of (4.1) with $f=\widehat{A}_{j} U$. Using (4.2), we have

$$
\left\|u_{j}\right\|_{H^{2}(\Omega)} \leq C\left\|\hat{A}_{j} U\right\|_{L^{2}(\Omega)},
$$

and using a standard finite element error estimate [13], we have

$$
\left\|u_{j}-U\right\|_{H^{1}(\Omega)} \leq C h_{j}\left\|u_{j}\right\|_{H^{2}(\Omega)} .
$$


By approximation properties of finite element spaces [13], we may choose a function $U^{I} \in M_{j}$ (e.g., the interpolant of $u_{j}$ at the nodes) such that

$$
\left\|u_{j}-U^{I}\right\|_{H^{1}\left(\tau_{j}^{i}\right)} \leq C h_{j}\left\|u_{j}\right\|_{H^{2}\left(\tau_{j}^{i}\right)} \quad \forall \tau_{j}^{i} \in T_{j} .
$$

Furthermore, it follows from (A.7) in [20] that

$$
\left\|U^{I}\right\|_{H^{2}\left(\tau_{j}^{i}\right)} \leq C\left\|u_{j}\right\|_{H^{2}\left(\tau_{j}^{i}\right)} \quad \forall \tau_{j}^{i} \in T_{j} .
$$

Since the mesh is quasi-uniform, we have the following inverse inequalities [13]:

$$
\|V\|_{H^{s}\left(\tau_{j}^{i}\right)} \leq C h_{j}^{r-s}\|V\|_{H^{r}\left(\tau_{j}^{i}\right)} \quad \forall V \in M_{j}, 0 \leq r \leq s \leq k .
$$

Combine (4.19)-(4.21) and the arithmetic-geometric mean inequality:

$$
\begin{aligned}
\|U\|_{H^{k}\left(\tau_{j}^{i}\right)}^{2} & \leq C h_{j}^{-2(k-2)}\left(\left\|U-U^{I}\right\|_{H^{2}\left(\tau_{j}^{i}\right)}^{2}+\left\|U^{I}\right\|_{H^{2}\left(\tau_{j}^{i}\right)}^{2}\right) \\
& \leq C h_{j}^{-2(k-2)}\left(h_{j}^{-2}\left\|U-U^{I}\right\|_{H^{1}\left(\tau_{j}^{i}\right)}^{2}+\left\|u_{j}\right\|_{H^{2}\left(\tau_{j}^{i}\right)}^{2}\right) \\
& \leq C h_{j}^{-2(k-2)}\left(h_{j}^{-2}\left\|u_{j}-U\right\|_{H^{1}\left(\tau_{j}^{i}\right)}^{2}+\left\|u_{j}\right\|_{H^{2}\left(\tau_{j}^{i}\right)}^{2}\right) .
\end{aligned}
$$

Now combine (4.17) and (4.18) with (4.22) and sum over all triangles $\tau_{j}^{i}$ :

$$
\sum_{\tau_{j}^{i} \in T_{j}}\|U\|_{H^{k}\left(\tau_{j}^{i}\right)}^{2} \leq C h_{j}^{-2(k-2)}\left\|\widehat{A}_{j} U\right\|_{L^{2}(\Omega)}^{2} .
$$

(4.15b) now follows from (4.16) and (4.23).

The proof of $(4.15 \mathrm{c})$ follows along the same lines as that of $(4.15 \mathrm{~b})$. In this case, $u_{j}$ is taken to be the solution of (4.1) with $f=A_{j} U$. Hence, $U$ is the discrete approximation of $u_{j}$ with the stiffness matrix approximated using numerical integration. $\widehat{A_{j}} U$ is now replaced by $A_{j} U$ in (4.17). Furthermore, error estimate (4.18) now follows from the proof of Theorem 4.1.6 in [13] (i.e., optimal energy estimates hold when (4.5) holds with $L=0)$. The remainder of the proof of $(4.15 \mathrm{c})$ is the same as that of $(4.15 \mathrm{~b})$.

We next prove two simple consequences of Lemma 4.4. Note that the injection operator will often be omitted when there is no danger of confusion.

Lemma 4.5. Suppose that the hypotheses of Lemma 4.4 hold. Then

$$
C^{-1}\left\|A_{j} U\right\|_{L^{2}(\Omega)} \leq\left\|\hat{A}_{j} U\right\|_{L^{2}(\Omega)} \leq C\left\|A_{j} U\right\|_{L^{2}(\Omega)} \quad \forall U \in M_{j}
$$

and

$$
C^{-1} \lambda_{j}^{-1}\left\|A_{j} U\right\|_{L^{2}(\Omega)} \leq \hat{\lambda}_{j}^{-1}\left\|\hat{A}_{j} U\right\|_{L^{2}(\Omega)} \leq C \lambda_{j}^{-1}\left\|A_{j} U\right\|_{L^{2}(\Omega)} \quad \forall U \in M_{j}
$$

Proof. In view of (4.12), it is clear that (4.24b) follows from (4.24a). To prove the first inequality in $(4.24 \mathrm{a})$, note that by the triangle inequality and Lemma 4.4 ,

$$
\left|A_{j}(U, V)\right| \leq\left|\widehat{A_{j}}(U, V)\right|+C h_{j}\|V\|_{H^{1}(\Omega)}\left\|\widehat{A_{j}} U\right\|_{L^{2}(\Omega)} \quad \forall U, V \in M_{j} .
$$


Also note that

$$
\left\|A_{j} U\right\|_{L^{2}(\Omega)}=\sup _{0 \neq v \in L^{2}(\Omega)} \frac{\left|\left(A_{j} U, v\right)\right|}{\|v\|_{L^{2}(\Omega)}} \leq \sup _{0 \neq v \in L^{2}} \frac{\left|\left(A_{j} U, V\right)\right|}{\|V\|_{L^{2}(\Omega)}},
$$

where $V$ is the $L^{2}$ projection of $v$ into $M_{j}$. Hence,

$$
\left\|A_{j} U\right\|_{L^{2}(\Omega)}=\sup _{0 \neq V \in M_{j}} \frac{\left|A_{j}(U, V)\right|}{\|V\|_{L^{2}(\Omega)}} .
$$

Combining this and (4.21) (with $s=1$ and $r=0$ ),

$$
\begin{aligned}
\left\|A_{j} U\right\|_{L^{2}(\Omega)} & \leq \sup _{0 \neq V \in M_{j}} \frac{\left|\widehat{A}_{j}(U, V)\right|}{\|V\|_{L^{2}(\Omega)}}+C^{\prime}\left\|\widehat{A}_{j} U\right\|_{L^{2}(\Omega)} \\
& \leq\left(C^{\prime}+1\right)\left\|\widehat{A}_{j} U\right\|_{L^{2}(\Omega)} .
\end{aligned}
$$

This proves the first estimate in (4.24a). The second estimate follows in the same way.

Lemma 4.6. Suppose that the hypotheses of Lemma 4.4 hold for both $M_{j}$ and $M_{j-1}$. Then for each $U \in M_{j}$,

$$
\left\|\left(\widehat{P}_{j-1}-P_{j-1}\right) U\right\|_{A_{j-1}} \leq C h_{j}^{L+1}\|U\|_{H^{1}(\Omega)} \text { if } k=1
$$

and

$$
\begin{aligned}
\left\|\left(\hat{P}_{j-1}-P_{j-1}\right) U\right\|_{A_{j-1}} & \leq C h_{j}^{L+2}\left\|\hat{A}_{j} U\right\|_{L^{2}(\Omega)} \\
& \leq C h_{j}^{L+2}\left\|A_{j} U\right\|_{L^{2}(\Omega)} \quad \text { if } k>1 .
\end{aligned}
$$

Proof. Using (4.8), (4.9), and the triangle inequality, we obtain

$$
\begin{aligned}
\mid A_{j-1} & \left(\left(\widehat{P}_{j-1}-P_{j-1}\right) U, V\right) \mid \\
\leq & \left|A_{j-1}\left(\widehat{P}_{j-1} U, V\right)-\widehat{A}\left(\widehat{P}_{j-1} U, V\right)\right| \\
& +\left|\widehat{A}(U, V)-A_{j}(U, V)\right| \quad \forall U \in M_{j}, \quad V \in M_{j-1} .
\end{aligned}
$$

Combining this with (4.11) and (4.15a), we deduce for $k=1$ :

$$
\begin{aligned}
\left\|\left(\widehat{P}_{j-1}-P_{j-1}\right) U\right\|_{A_{j-1}} & =\sup _{0 \neq V \in M_{j-1}} \frac{\left|A_{j-1}\left(\left(\widehat{P}_{j-1}-P_{j-1}\right) U, V\right)\right|}{\|V\|_{A_{j-1}}} \\
& \leq C h_{j}^{L+1}\left(\left\|\widehat{P}_{j-1} U\right\|_{\widehat{A}}+\|U\|_{H^{1}(\Omega)}\right) \leq C h_{j}^{L+1}\|U\|_{H^{1}(\Omega)},
\end{aligned}
$$

using the fact that $\widehat{P}_{j-1}$ is a projection operator with respect to $\widehat{A}($,$) in the$ last step. This proves $(4.25 \mathrm{a})$.

Similarly, we may combine (4.11), (4.15b), and (4.26) to deduce for $k>1$ :

$$
\left\|\left(\widehat{P}_{j-1}-P_{j-1}\right) U\right\|_{A_{j-1}} \leq C h_{j}^{L+2}\left(\left\|\widehat{A}_{j} U\right\|_{L^{2}(\Omega)}+\left\|\widehat{A}_{j-1} \widehat{P}_{j-1} U\right\|_{L^{2}(\Omega)}\right) \text {. }
$$


To estimate the last term on the right, note that it readily follows, using (4.9), that

$$
\widehat{A}_{j-1} \widehat{P}_{j-1}=P_{j-1}^{0} \widehat{A}_{j},
$$

where $P_{j-1}^{0}$ denotes the $L^{2}$ projection from $M_{j}$ into $M_{j-1}$. Hence,

$$
\left\|\widehat{A}_{j-1} \widehat{P}_{j-1} U\right\|_{L^{2}(\Omega)}=\left\|P_{j-1}^{0} \widehat{A}_{j} U\right\|_{L^{2}(\Omega)} \leq\left\|\widehat{A}_{j} U\right\|_{L^{2}(\Omega)} .
$$

Combining this with (4.27), we obtain the first estimate in $(4.25 \mathrm{~b})$. The second estimate follows using (4.24a).

We now show that conditions (A.4)-(A.7) hold.

Theorem 4.1. Suppose that the hypotheses of Lemma 4.4 hold for each $j=$ $1, \ldots, J$. Then conditions (A.4)-(A.7) hold.

(a) If $k=1$ and $L=0$, then $\alpha=1 / 2$ in (A.4). Otherwise, $\alpha=1$.

(b) If $k=1$, then $\sigma=(L+1) / 2$ in (3.3a) and (3.12a) for $L \geq 0$ in (4.5).

Furthermore, $\sigma=L / 2$ in (3.3b) and (3.12b) if $L>0$ in (4.5).

(c) If $k>1$, then $\sigma=(L+1) / 2$ in (3.3b) and (3.12b) for $L \geq 0$ in (4.5).

Proof. Note that

$$
\begin{aligned}
\left\|\widehat{P}_{j-1} U\right\|_{H^{1}(\Omega)} & \leq C\|U\|_{H^{1}(\Omega)}, \\
\left\|P_{j-1} U\right\|_{H^{1}(\Omega)} & \leq C\|U\|_{H^{1}(\Omega)} .
\end{aligned}
$$

(4.28a) is immediate from (4.11), since $\widehat{P}_{j-1}$ is an orthogonal projection with respect to $\widehat{A}($,$) . (4.28b) follows easily, using (4.11), (4.28a), and Lemma 4.6.$

(a) Suppose that $k=1$ and $U \in M_{j}$. Using (4.15a) and (4.28b), we have

$$
\left|A_{j}\left(\left(I-P_{j-1}\right) U, U\right)-\widehat{A}\left(\left(I-P_{j-1}\right) U, U\right)\right| \leq C h_{j}^{L+1}\|U\|_{H^{1}(\Omega)}^{2} .
$$

(4.29) and the triangle inequality yield

$$
\begin{aligned}
\left|A_{j}\left(\left(I-P_{j-1}\right) U, U\right)\right| \leq & C h_{j}^{L+1}\|U\|_{H^{1}(\Omega)}^{2}+\left|\widehat{A}\left(\left(\widehat{P}_{j-1}-P_{j-1}\right) U, U\right)\right| \\
& +\left|\widehat{A}\left(\left(I-\widehat{P}_{j-1}\right) U, U\right)\right| .
\end{aligned}
$$

Apply (4.11) and (4.25a) to the second term on the right and apply (A.3) and $(4.24 \mathrm{~b})$ to the last term. Hence,

$$
\left|A_{j}\left(\left(I-P_{j-1}\right) U, U\right)\right| \leq C h_{j}^{L+1}\|U\|_{A_{j}}^{2}+C_{1} \lambda_{j}^{-1}\left\|A_{j} U\right\|_{j}^{2} \quad \text { if } k=1 .
$$

Combining (3.7), (4.12), and (4.30), we see that (A.4) holds with $\alpha=1 / 2$ if $L=0$ and $\alpha=1$ if $L \geq 1$. Finally, if $k>1$ and $L \geq 0$, we apply the preceding argument, replacing (4.15a) and (4.25a) by $(4.15 \mathrm{c})$ and $(4.25 \mathrm{~b})$. Hence,

$$
\begin{aligned}
\left|A_{j}\left(\left(I-P_{j-1}\right) U, U\right)\right| \leq & C h_{j}^{L+2}\|U\|_{A_{j}}\left\|A_{j} U\right\|_{L^{2}} \\
& +C_{1} \lambda_{j}^{-1}\left\|A_{j} U\right\|_{L^{2}}^{2} \text { if } k>1 .
\end{aligned}
$$

In view of (3.7), (4.12), and (4.31), we see that $\alpha=1$ in (A.4) holds if $k>1$. 
(b) It is immediate from (4.12) and (4.30) that (A.6) holds for $L \geq 0$ with $\sigma=(L+1) / 2$ in (3.3a). If $L>0$, apply (3.7) and (4.12) to the first term on the right side of (4.30) to see that $\sigma=L / 2$ in (3.3b). To prove (A.7), we again apply (4.11), (4.15a), (4.25a), (4.28), and the triangle inequality as in the proof of (4.30) to deduce $\forall U, V \in M_{j-1}$,

$$
\begin{aligned}
\left|A_{j-1}\left(\left(I-P_{j-1} I_{j}\right) U, V\right)\right| \leq & C h_{j}^{L+1}\|U\|_{A_{j-1}(\Omega)}\|V\|_{A_{j-1}(\Omega)} \\
& +\widehat{A}\left(\left(I-\widehat{P}_{j-1} I_{j}\right) U, V\right) .
\end{aligned}
$$

Since $\widehat{P}_{j-1}$ is an orthogonal projection operator, the last term in (4.32) vanishes. Now combine (4.12) and (4.32) to see that (A.7) holds with $\sigma=(L+1) / 2$ in (3.12a) for $L \geq 0$. It can be seen, using analogous arguments, that (A.5) holds with $\sigma=(L+1) / 2$ in (3.3a). Alternatively, (A.5) follows from (A.7) and Lemma A.1 in the Appendix. Finally, it is immediate from the preceding results and (3.7) that (A.5) and (A.7) hold with $\sigma=L / 2$ in (3.3b) and (3.12b) if $L>0$. This completes the proof of (b).

(c) The proof is similar to that of (b) with (4.15a), (4.25a), and (4.30) replaced by (4.15c), (4.25b), and (4.31), respectively.

4.2. Multigrid estimates. We now prove a number of multigrid results for the variable $\mathscr{V}$ cycle (see (2.6b)) and $\mathscr{V}$ cycle (see (2.6a)). We first consider the symmetric operator $B_{J}^{S}$.

Theorem 4.2. Suppose that (A.1) and the hypotheses of Lemma 4.4 hold for $j=1, \ldots, J$.

(a) If (2.6b) holds with $m_{J} \geq 1$, then

$$
\kappa\left(B_{J}^{S} A_{J}\right)=O(1) \text { as } J \rightarrow \infty .
$$

(b) If (2.6a) holds with $m_{J} \geq 1$, then

(4.33b) $\quad \kappa\left(B_{J}^{S} A_{J}\right)=O(1+J)=O\left(\left|\log h_{J}\right|\right) \quad$ as $J \rightarrow \infty$ for $k=1$ and $L=0$.

Otherwise, (4.33a) holds.

Proof. (a) This follows immediately from Theorem 3.1 and Theorem 4.1(a).

(b) This follows from Theorem 3.2 and Theorem 4.1(a).

It follows from Theorem 4.2 that $B_{J}^{S}$ can be used effectively as a preconditioner for conjugate gradient and other iterative methods, even with $m_{J}=1$. In Theorem 4.3S below, we show that $I-B_{J}^{S} A_{J}$ is a contraction operator with contraction number independent of $h$ and $J$, assuming a suitable constraint relating $L, m_{J}, h_{0}$, and $h_{J}$. For simplicity, we assume that for the variable $\mathscr{V}$ cycle,

$$
m_{j-1}=2 m_{j}, \quad j=2, \ldots, J .
$$

The more general situation expressed in $(2.6 \mathrm{~b})$ can be treated in the same way. Set $h \equiv h_{J}$ and $m \equiv m_{J} \geq 1$. 
Theorem 4.3S. Assume that (A.1) and the hypotheses of Lemma 4.4 hold for $j=1, \ldots, J$. Define $\bar{k} \equiv 1$ if $k>1$ and $\bar{k} \equiv 0$ if $k=1$.

(a) Suppose that $B_{J}^{S}$ is a variable $\mathscr{V}$ cycle and (4.34) holds. Then

$$
-\delta_{J}^{(1)}\|U\|_{A_{J}}^{2} \leq A_{J}\left(\left(I-B_{J}^{S} A_{J}\right) U, U\right) \leq \delta_{J}^{(2)}\|U\|_{A_{J}}^{2}
$$

with $\delta_{J}^{(1)}$ and $\delta_{J}^{(2)}$ defined as follows. If $k>1$ or $L>0$, then

$$
\delta_{J}^{(1)}=C(\varepsilon) h_{0}^{L-1+k} h^{(2 / 3-\varepsilon)} m^{-(2 / 3+\varepsilon / 2)} \quad \forall \varepsilon \in(0,2 / 3)
$$

and

$$
\delta_{J}^{(2)}=\delta \equiv \frac{C_{0}}{C_{0}+m} .
$$

If $k=1$ and $L=0$, then

$$
\delta_{J}^{(1)}=C(\varepsilon) h^{1 / 3-\varepsilon} m^{-(1 / 3+\varepsilon / 2)} \quad \forall \varepsilon \in(0,1 / 3)
$$

and

$$
\delta_{J}^{(2)}=\min \left(\delta+\delta_{J}^{(1)}, \frac{C_{0}}{C_{0}+m^{1 / 2}}\right) .
$$

$C_{0}$ and $C(\varepsilon)$ are independent of $h_{0}, J$, and $m$. Furthermore,

$$
\left|A_{J}\left(\left(I-B_{J}^{S} A_{J}\right) U, U\right)\right| \leq \delta_{J}\|U\|_{A_{J}}^{2} \equiv \max \left(\delta_{J}^{(1)}, \delta_{J}^{(2)}\right)\|U\|_{A_{J}}^{2} .
$$

(b) Suppose that $B_{J}^{S}$ is a $\mathscr{V}$ cycle (i.e., (2.6a) holds). Then (4.35) and (4.37) hold. $\delta_{J}^{(1)}$ is given by (4.36a) or (4.36c) with $h$ replaced by $h_{0}$. Furthermore, $\delta_{J}^{(2)}$ is given by (4.36b) if $k>1$ or $L>0$. If $k=1$ and $L=0$, then (4.36d) is replaced by

$$
\delta_{J}^{(2)}=\min \left(\delta+\delta_{J}^{(1)}, \frac{C_{0} J}{C_{0} J+m^{1 / 2}}\right) .
$$

Before proving Theorem 4.3S, we make some observations.

Remark 4.2. The exponent of $m$ in $(4.36 \mathrm{a}, \mathrm{c})$ is clearly not uniquely determined, nor is it limited to the values given in these expressions. The main point of Theorem 4.3S is that $I-B_{J}^{S} A_{J}$ has a fixed error reduction, independent of $h$ and $J$, when $\max \left(\delta_{J}^{(1)}, \delta_{J}^{(2)}\right)<1$. It follows from (a) for the variable $\mathscr{V}$ cycle that

$$
\delta_{J} \rightarrow \delta \text { as } m \rightarrow \infty \text { or } h \rightarrow 0,
$$

where $\delta$ in (4.36b) is the same as in Theorem 2.1. Hence, less smoothings may be required for convergence for smaller values of $h$ (i.e., larger computational problems). It follows from (b) for the $\mathscr{V}$ cycle that

$$
\delta_{J} \rightarrow \delta \text { as } m \rightarrow \infty \text { or } h_{0} \rightarrow 0 .
$$


Remark 4.3. The number of smoothings required for $I-B_{J}^{S} A_{J}$ to be a contraction can be further reduced if a more accurate quadrature scheme is used on coarse grid levels. For example, suppose the quadrature scheme is exact on $M_{j}$ for $j=1, \ldots, J_{0}$, where $J_{0}=J-j_{0}$ with $j_{0} \geq 1$ independent of $J$. (If the coefficients in (4.1a) are constant, the quadrature is exact when $L=2$ in (4.5).) We can now replace $h_{0}$ by $h_{J_{0}}$ (and hence $\left.h\right)$ in (4.36a) and (4.36c) for both the $\mathscr{V}$ and variable $\mathscr{V}$ cycles. This can be seen from the proof of Theorem 4.3S.

Proof of Theorem 4.3S. (a) For the first estimate in (4.35), we apply Theorem 3.3. First assume that $k>1$. By Theorem 4.1(c), we see that (A.5) holds with $\sigma=(L+1) / 2$ in (3.3b). Applying (4.12) and (3.9c) with $\sigma_{0}=1 / 6+\varepsilon / 2$, we obtain

$$
\begin{aligned}
\eta_{1} & \leq \prod_{j=1}^{J}\left(1+C h_{j}^{L} h_{j}^{2 / 3-\varepsilon} m_{j}^{-(2 / 3+\varepsilon / 2)}\right) \\
& \leq \prod_{j=0}^{J-1}\left(1+C h_{0}^{L}\left(2^{j}\right)^{-3 \varepsilon / 2} h^{2 / 3-\varepsilon} m^{-(2 / 3+\varepsilon / 2)}\right),
\end{aligned}
$$

using (4.4) and (4.34) in the last step. Using (4.38), we conclude after a simple calculation:

$$
\eta_{1} \leq 1+C(\varepsilon) h_{0}^{L} h^{2 / 3-\varepsilon} m^{-(2 / 3+\varepsilon / 2)} .
$$

The first estimate in (4.35) with $\delta_{J}^{(1)}$ in (4.36a) (with $\bar{k}=1$ ) thus follows from (3.9a) and (4.39). If $k=1$, it follows analogously, using Theorem 4.1(b), that $\delta_{J}^{(1)}$ is given by (4.36a) with $\bar{k}=0$ if $L>0$ and by (4.36c) if $L=0$. (In the last case, we use (3.9b) with $\sigma=1 / 2$ and $\sigma_{0}=1 / 3+\varepsilon / 2$.)

Next consider the second estimate in (4.35). It follows from Theorem 4.1(a) that $\alpha=1$ in (A.4) if $k>1$ or $L>0$. (4.36b) thus follows from (3.8a) and (3.8c) (with $\alpha=1$ ) in Theorem 3.3. If $k=1$ and $L=0$, then $\alpha=1 / 2$ in (A.4) by Theorem $4.1(\mathrm{a})$. Hence, $\delta_{J}^{(2)}$ is bounded by $C_{0} /\left(C_{0}+m^{1 / 2}\right)$, using (3.8a) and (3.8c). To complete the proof of (4.36d), it thus suffices to prove

$$
\delta_{J}^{(2)} \leq \delta+C(\varepsilon) h^{1 / 3-\varepsilon} m^{-(1 / 3+\varepsilon / 2)} .
$$

To prove (4.40), we apply $(3.16 \mathrm{a}, \mathrm{b})$ in Theorem $3.4 \mathrm{~S}$. It follows from Theorem 4.1(b) that $\sigma=1 / 2$ in (3.3a). Now combine (4.12) and (3.16b) (with $\sigma_{0}=$ $1 / 3+\varepsilon / 2)$ to deduce

$$
\delta_{J}^{(2)} \leq \delta+C \sum_{j=1}^{J} h_{j}^{1 / 3-\varepsilon} m_{j}^{-(1 / 3+\varepsilon / 2)} \leq \delta+C \sum_{j=0}^{J-1}\left(2^{j}\right)^{-3 \varepsilon / 2} h^{1 / 3-\varepsilon} m^{-(1 / 3+\varepsilon / 2)},
$$

using (4.4) and (4.34) in the last step. (4.40) follows readily from (4.41). Finally, (4.37) is an immediate consequence of (4.35). This completes the proof of (a). 
(b) The proof is analogous to that of (a) and need not be repeated. Note that $\delta_{J}^{(2)}$ is now estimated using $(3.8 \mathrm{~b})$ instead of $(3.8 \mathrm{c})$. Hence we replace $(4.36 \mathrm{~d})$ by $(4.36 \mathrm{e})$.

We next prove an analogue of Theorem 4.3S for the nonsymmetric multigrid operator $B_{J}^{N}$ using Theorem 3.3N. Note that the observations in Remarks 4.2 and 4.3 are also applicable in this case.

Theorem 4.3N. Assume that (A.1) and the hypotheses of Lemma 4.4 hold for $j=1, \ldots, J$.

(a) Suppose that $B_{J}^{N}$ is a variable $\mathscr{V}$ cycle and (4.34) holds. Then

$$
\left\|\left(I-B_{J}^{N} A_{J}\right) U\right\|_{A_{J}}^{2} \leq \delta_{J}\|U\|_{A_{J}}^{2}
$$

with

$$
\delta_{J}=\delta+C(\varepsilon) h_{0}^{L} h^{1 / 3-\varepsilon} m^{-(1 / 3+\varepsilon / 2)} \quad \forall \varepsilon \in(0,1 / 3),
$$

where $\delta$ is given by (4.36b) and $C(\varepsilon)$ is independent of $h_{0}, J$, and $m$.

(b) Suppose that $B_{J}^{N}$ is a $\mathscr{V}$ cycle. Then (4.42a) holds with $h$ replaced by $h_{0}$ in $(4.42 \mathrm{~b})$.

Proof. (a) This follows from the same argument as in the proof of (4.40) in Theorem 4.3S. In the present case, we apply Theorem $3.4 \mathrm{~N}$ (with $\sigma=(L+1) / 2$ and $\sigma_{0}=1 / 3+\varepsilon / 2$ in $\left.(3.13 b)\right)$.

(b) The proof is analogous to that of (a).

Remark 4.4. The numerical quadrature analysis for three-dimensional problems goes through in the same way as for two-dimensional problems. Hence, analogues of the multigrid results in this section also hold. Note that the system of equations resulting from finite difference discretizations do not typically fall in the variational multigrid framework. However, it is known that many finite difference schemes in two and three dimensions can be obtained by applying the finite element method with a suitable numerical quadrature. We refer to [13] (see the exercises at the end of §4.1) and [14] (see §4.3) for several examples illustrating this. Hence, multigrid estimates for these finite difference schemes also follow from the preceding results. In particular, standard secondorder centered difference schemes can often be obtained from a finite element method with piecewise linear elements and a quadrature scheme that is exact on constants (i.e., $k=1$ and $L=0$ in (4.5)).

\section{APPENDIX}

We prove Theorems $3.3,3.4 \mathrm{~N}$, and $3.4 \mathrm{~S}$ of $\S 3$. We begin with

Lemma A.1. Suppose that $C^{-1} \lambda_{j-1}^{-1} \leq \lambda_{j}^{-1} \leq C \lambda_{j-1}^{-1}$. Then

(a) (3.3b) implies (3.3a), and (3.12b) implies (3.12a);

(b) (3.12a) implies (3.3a). 
Proof. (a) Since $A_{j}$ is positive definite symmetric, it follows, using (2.1a), that

$$
\left\|A_{j} U\right\|_{j} \leq\left\|A_{j}^{1 / 2}\right\|\left\|_{j} \cdot\right\| A_{j}^{1 / 2} U\left\|_{j}=\lambda_{j}^{1 / 2}\right\| U \|_{A_{j}} \quad \forall U \in M_{j},
$$

where ||$\cdot \mid \|_{j}$ denotes the operator norm on $M_{j}$. The proof of (a) follows immediately from (1).

(b) (3.12a) is equivalent to

$$
\left|A_{j-1}\left(\left(I-P_{j-1} I_{j}\right) U, V\right)\right| \leq C_{2} \lambda_{j}^{-\sigma}\|U\|_{A_{j-1}}\|V\|_{A_{j-1}} \quad \forall V \in M_{j-1} .
$$

Setting $V=U$, we obtain

$$
\left\|I_{j} U\right\|_{A_{j}}^{2} \leq\|U\|_{A_{j-1}}^{2}+C_{2} \lambda_{j}^{-\sigma}\|U\|_{A_{j-1}}^{2} \quad \forall U \in M_{j-1} .
$$

Define the operator norms of $I_{j}$ and $P_{j-1}$ by

$$
\left\|P_{j-1} \mid\right\|_{A_{j-1}} \equiv \max _{0 \neq U \in M_{j}} \frac{\left\|P_{j-1} U\right\|_{A_{j-1}}}{\|U\|_{A_{j}}}
$$

and

$$
\left\|\mid I_{j}\right\|_{A_{j}} \equiv \max _{0 \neq U \in M_{j-1}} \frac{\left\|I_{j} U\right\|_{A_{j}}}{\|U\|_{A_{j-1}}} .
$$

Since $P_{j-1}$ and $I_{j}$ are adjoints, their operator norms are equal. Hence, it follows from $(2 b)$ that

$$
\left\|I_{j}\left|\left\|_{A_{j}}^{2} \equiv\right\|\right|\left|P_{j-1}\right|\right\|_{A_{j-1}}^{2} \leq 1+C_{2} \lambda_{j}^{-\sigma} .
$$

(3.3a) now follows from (3).

We also use the following lemma, proved in [16, Lemma 4.1].

Lemma A.2. Suppose that $B_{J}^{S}$ is the symmetric $\mathscr{V}$ or variable $\mathscr{V}$ cycle given by Definition 2.1 and that $\bar{\delta}_{j}$ for $j=1, \ldots, J$ satisfies

(4) $-A_{j}\left(\left(I-I_{j} P_{j-1}\right) K_{j}^{m_{j}} U, K_{j}^{m_{j}} U\right) \leq \bar{\delta}_{j}\|U\|_{A_{j}}^{2} \quad \forall U \in M_{j}, j=1, \ldots, J$.

Then the constant $\eta_{1}$ in (3.1) satisfies

$$
\eta_{1} \leq \prod_{j=1}^{J}\left(1+\bar{\delta}_{j}\right)
$$

Proof of Theorem 3.3. Estimates (3.8a), (3.8b) follow using (3.1) and (3.5). (3.8a), (3.8c) follow from (3.1) and (3.2a). We next prove (3.9a), (3.9b), using Lemma A.2. If (3.3a) holds, it follows readily, using (3.7), that for each $U \in$ $M_{j}$,

$$
\begin{aligned}
& -A_{j}\left(\left(I-I_{j} P_{j-1}\right) K_{j}^{m_{j}} U, K_{j}^{m_{j}} U\right) \\
& \quad \leq C_{2} C_{3}^{\sigma_{0}} \lambda_{j}^{-\sigma}\left\|A_{j} K_{j}^{m_{j}} U\right\|_{j}^{2 \sigma_{0}} \cdot\left\|K_{j}^{m_{j}} U\right\|_{A_{j}}^{2-2 \sigma_{0}} .
\end{aligned}
$$


Combining the last estimate with (2.8), (3.1), Lemma 2.1, and Lemma A.2, we obtain (3.9a) and (3.9b). The inequality in (3.9b) follows using (3.4), since each $m_{j} \geq m_{J}$. The proof of $(3.9 \mathrm{a}),(3.9 \mathrm{c})$ is essentially the same. It is immediate from (3.8a) and (3.9a) that (3.10a) holds with $\delta_{J}$ given by $(3.10 \mathrm{~b})$. Finally, it follows from $(3.8 \mathrm{~b}, \mathrm{c}),(3.9 \mathrm{~b}, \mathrm{c})$, and $(3.10 \mathrm{~b})$ that $\delta_{J}<1$ for $m_{J}$ sufficiently large.

In the remainder, we set $m \equiv m_{J}$. The proof of Theorem $3.4 \mathrm{~N}$ is based on the following lemma.

Lemma A.3N. Suppose that (A.1), (A.6), and (A.7) hold. Also assume that for some $J \geq 1$ and $\tilde{\delta}_{J-1}$, we have

$$
\left\|\left(I-B_{J-1}^{N} A_{J-1}\right) U\right\|_{A_{J-1}}^{2} \leq\left(\delta+\tilde{\delta}_{J-1}\right)\|U\|_{A_{J-1}}^{2} \quad \forall U \in M_{J-1},
$$

where $\delta$ is defined by $(3.14 \mathrm{a}, \mathrm{b})$. Then

$$
\left\|\left(I-B_{J}^{N} A_{J}\right) U\right\|_{A_{J}}^{2} \leq \delta\|U\|_{A_{J}}^{2}+\left(\tilde{\delta}_{J-1}+\tilde{\delta}_{J}\right)\left\|K_{J}^{m} U\right\|_{A_{J}}^{2} \quad \forall U \in M_{J},
$$

where

$$
\tilde{\delta}_{J} \leq C_{2} \lambda_{J}^{-\sigma}\left(4+3 \tilde{\delta}_{J-1}+4 C_{2} \lambda_{J}^{-\sigma}+2 C_{2} \lambda_{J}^{-\sigma} \tilde{\delta}_{J-1}\right)
$$

Proof. It follows from Lemma A.1 that (A.5) and (A.6) hold with $E_{j}$ given by (3.3a), and that $F_{j}$ in (A.7) is given by $(3.12 \mathrm{a})$. Applying $(2.7 \mathrm{~N})$, we have

$$
\begin{aligned}
E \equiv & \left\|\left(I-B_{J}^{N} A_{J}\right) U\right\|_{A_{J}}^{2} \\
= & \left\|\left(I-I_{J} P_{J-1}\right) K_{J}^{m} U\right\|_{A_{J}}^{2} \\
& +\left\|I_{J}\left(I-B_{J-1}^{N} A_{J-1}\right) P_{J-1} K_{J}^{m} U\right\|_{A_{J}}^{2} \\
& +2 A_{J}\left(\left(I-I_{J} P_{J-1}\right) K_{J}^{m} U, I_{J}\left(I-B_{J-1}^{N} A_{J-1}\right) P_{J-1} K_{J}^{m} U\right) .
\end{aligned}
$$

We apply (3) to deduce

$$
\begin{aligned}
\left\|\left(I-I_{J} P_{J-1}\right) K_{J}^{m} U\right\|_{A_{J}}^{2} \leq & A_{J}\left(\left(I-I_{J} P_{J-1}\right) K_{J}^{m} U, K_{J}^{m} U\right) \\
& +C_{2} \lambda_{J}^{-\sigma}\left\|P_{J-1} K_{J}^{m} U\right\|_{A_{J-1}}^{2} \\
\leq & A_{J}\left(\left(I-I_{J} P_{J-1}\right) K_{J}^{m} U, K_{J}^{m} U\right) \\
& +C_{2} \lambda_{J}^{-\sigma}\left(1+C_{2} \lambda_{J}^{-\sigma}\right)\left\|K_{J}^{m} U\right\|_{A_{J}}^{2}
\end{aligned}
$$

To estimate the second term on the right side of (9), we combine (3) and (6) to obtain

$$
\begin{aligned}
& \left\|I_{J}\left(I-B_{J-1}^{N} A_{J-1}\right) P_{J-1} K_{J}^{m} U\right\|_{A_{J}}^{2} \\
& \leq\left(1+C_{2} \lambda_{J}^{-\sigma}\right)\left(\delta+\tilde{\delta}_{J-1}\right)\left\|P_{J-1} K_{J}^{m} U\right\|_{A_{J}}^{2} \\
& \leq \delta\left\|P_{J-1} K_{J}^{m} U\right\|_{A_{J}}^{2}+\left(\delta C_{2} \lambda_{J}^{-\sigma}\left(1+C_{2} \lambda_{J}^{-\sigma}\right)\right. \\
& \left.\quad+\tilde{\delta}_{J-1}\left(1+C_{2} \lambda_{J}^{-\sigma}\right)^{2}\right)\left\|K_{J}^{m} U\right\|_{A_{J}}^{2}
\end{aligned}
$$


We also use the identity

$$
\begin{aligned}
& A_{J}\left(\left(I-I_{J} P_{J-1}\right) V, V\right)+\delta\left\|P_{J-1} V\right\|_{A_{J-1}}^{2} \\
& \quad=(1-\delta) A_{J}\left(\left(I-I_{J} P_{J-1}\right) V, V\right)+\delta\|V\|_{A_{J}}^{2} \quad \forall V \in M_{J} .
\end{aligned}
$$

We may now apply (9)-(12) and the symmetry of $K_{J}$ to see that

$$
\begin{aligned}
E \leq & (1-\delta) A_{J}\left(\left(I-I_{J} P_{J-1}\right) K_{J}^{m} U, K_{J}^{m} U\right)+\delta A_{J}\left(K_{J}^{2 m} U, U\right) \\
& +\left(1+C_{2} \lambda_{J}^{-\sigma}\right)\left((1+\delta) C_{2} \lambda_{J}^{-\sigma}+\tilde{\delta}_{J-1}\left(1+C_{2} \lambda_{J}^{-\sigma}\right)\right)\left\|K_{J}^{m} U\right\|_{A_{J}}^{2} \\
& +2 A_{J}\left(\left(I-I_{J} P_{J-1}\right) K_{J}^{m} U, I_{J}\left(I-B_{J-1}^{N} A_{J-1}\right) P_{J-1} K_{J}^{m} U\right) .
\end{aligned}
$$

To estimate the last term in (13), we apply inequality (2a) with $U$ replaced by $P_{J-1} K_{J}^{m} U$ and $V$ replaced by $\left(I-B_{J-1}^{N} A_{J-1}\right) P_{J-1} K_{J}^{m} U$. Hence,

$$
\begin{aligned}
\mid 2 A_{J} & \left.\left(I-I_{J} P_{J-1}\right) K_{J}^{m} U, I_{J}\left(I-B_{J-1}^{N} A_{J-1}\right) P_{J-1} K_{J}^{m} U\right) \mid \\
& \leq 2 C_{2} \lambda_{J}^{-\sigma}\left\|P_{J-1} K_{J}^{m} U\right\|_{A_{J-1}}\left\|\left(I-B_{J-1}^{N} A_{J-1}\right) P_{J-1} K_{J}^{m} U\right\|_{A_{J-1}} \\
& \leq C_{2} \lambda_{J}^{-\sigma}\left(1+C_{2} \lambda_{J}^{-\sigma}\right)\left(1+\delta+\tilde{\delta}_{J-1}\right)\left\|K_{J}^{m} U\right\|_{A_{J}}^{2},
\end{aligned}
$$

using (3), (6), and the arithmetic-geometric mean inequality in the last step. Next combine (13) and (14), and use the fact that $\delta<1$ (by (3.14a)). Thus,

$$
\begin{aligned}
E \leq & (1-\delta) A_{J}\left(\left(I-I_{J} P_{J-1}\right) K_{J}^{m} U, K_{J}^{m} U\right) \\
& +\delta A_{J}\left(K_{J}^{2 m} U, U\right)+\tilde{\delta}_{J-1}\left\|K_{J}^{m} U\right\|_{A_{J}}^{2} \\
& +C_{2} \lambda_{J}^{-\sigma}\left(3+\delta+3 \tilde{\delta}_{J-1}+4 C_{2} \lambda_{J}^{-\sigma}+2 C_{2} \tilde{\delta}_{J-1} \lambda_{J}^{-\sigma}\right)\left\|K_{J}^{m} U\right\|_{A_{J}}^{2}
\end{aligned}
$$

We now apply (A.6), with $E_{j}$ given by (3.3a), and Lemma 2.1 to estimate the first term on the right side of (15). Thus,

$$
\begin{aligned}
E \leq & C_{1} \widetilde{C}(2 m)^{-1}(1-\delta) A_{J}\left(\left(I-K_{J}^{2 m}\right) U, U\right) \\
& +\delta A_{J}\left(K_{J}^{2 m} U, U\right)+\tilde{\delta}_{J-1}\left\|K_{J}^{m} U\right\|_{A_{J}}^{2} \\
& +C_{2} \lambda_{J}^{-\sigma}\left(4+3 \tilde{\delta}_{J-1}+4 C_{2} \lambda_{J}^{-\sigma}+2 C_{2} \tilde{\delta}_{J-1} \lambda_{J}^{-\sigma}\right)\left\|K_{J}^{m} U\right\|_{A_{J}}^{2} .
\end{aligned}
$$

It follows from $(3.14 a)$ that

$$
C_{0}(m)^{-1}(1-\delta)=\delta \text {. }
$$

Combining (3.14b), (16), and (17), we obtain (7) and (8).

The proof of Theorem $3.4 \mathrm{~S}$ is based on the following lemma.

Lemma A.3S. Suppose that (A.1), (A.5), and (A.6) hold. Also assume that for some $J \geq 1$ and $\tilde{\delta}_{J-1}$ we have

$$
A_{J-1}\left(\left(I-B_{J-1}^{S} A_{J-1}\right) U, U\right) \leq\left(\delta+\tilde{\delta}_{J-1}\right)\|U\|_{A_{J-1}}^{2} \quad \forall U \in M_{J-1},
$$


where $\delta$ is defined by $(3.14 \mathrm{a}, \mathrm{b})$. Then

$$
A_{J}\left(\left(I-B_{J}^{S} A_{J}\right) U, U\right) \leq \delta\|U\|_{A_{J}}^{2}+\left(\tilde{\delta}_{J-1}+\tilde{\delta}_{J}\right)\left\|K_{J}^{m} U\right\|_{A_{J}}^{2},
$$

where

(20)

$$
\tilde{\delta}_{J} \leq C_{2} \lambda_{J}^{-\sigma}\left(1+\tilde{\delta}_{J-1}\right)
$$

Proof. Apply (2.7S) to obtain

$$
\begin{aligned}
A_{J}\left(\left(I-B_{J}^{S} A_{J}\right) U, U\right) \\
=A_{J}\left(\left(I-I_{J} P_{J-1}\right) K_{J}^{m} U, K_{J}^{m} U\right) \\
\quad+A_{J-1}\left(\left(I-B_{J-1}^{S} A_{J-1}\right) P_{J-1} K_{J}^{m} U, P_{J-1} K_{J}^{m} U\right) .
\end{aligned}
$$

The proof now goes through in the same way as that of Lemma A.3N. The details are less tedious in the present case, since the right side of $(21)$ is easier to estimate than that of (9). In particular, condition (A.7) was needed to estimate the last term in (9), but is not needed for (21).

We now prove Theorems $3.4 \mathrm{~S}$ and $3.4 \mathrm{~N}$.

Proof of Theorem 3.4S. Recall that (3.3b) implies (3.3a). We use an induction argument to prove (3.16a) with

$$
\delta^{(2)} \leq \delta+\left(\prod_{j=1}^{J}\left(1+C_{2} \lambda_{j}^{-\sigma}\right)\right) \cdot\left(\sum_{j=1}^{J}\left(C_{3}^{\sigma_{0}}\right)^{\eta} C_{2} \lambda_{j}^{-\left(\sigma-\sigma_{0}\right)}\left(\tilde{C} / 2 m_{j}\right)^{\sigma_{0}}\right),
$$

where $\eta=1$ if $C_{3}>1$ and $\eta=0$ if $C_{3} \leq 1$, with $C_{3}$ in (3.7). For $J=0$, this follows trivially, since $B_{0}=A_{0}^{-1}$. Next assume that $J>0$ and

$$
A_{J-1}\left(\left(I-B_{J-1}^{S} A_{J-1}\right) U, U\right) \leq\left(\delta+\tilde{\delta}_{J-1}\right)\|U\|_{A_{J-1}}^{2},
$$

with

$$
\tilde{\delta}_{J-1} \leq\left(\prod_{j=1}^{J-1}\left(1+C_{2} \lambda_{j}^{-\sigma}\right)\right) \cdot\left(\sum_{j=1}^{J-1}\left(C_{3}^{\sigma_{0}}\right)^{\eta} C_{2} \lambda_{j}^{-\left(\sigma-\sigma_{0}\right)}\left(\tilde{C} / 2 m_{j}\right)^{\sigma_{0}}\right) .
$$

Applying (2.8), Lemma A.3S, and (23a,b), we deduce

$$
\begin{aligned}
& A_{J}\left(\left(I-B_{J}^{S} A_{J}\right) U, U\right) \\
& \leq \delta\|U\|_{A_{J}}^{2}+\tilde{\delta}_{J-1}\left(1+C_{2} \lambda_{J}^{-\sigma}\right)\left\|K_{J}^{m} U\right\|_{A_{J}}^{2}+C_{2} \lambda_{J}^{-\sigma}\left\|K_{J}^{m} U\right\|_{A_{J}}^{2} \\
& \leq\left(\delta+\prod_{j=1}^{J}\left(1+C_{2} \lambda_{j}^{-\sigma}\right) \cdot\left(\sum_{j=1}^{J-1}\left(C_{3}^{\sigma_{0}}\right)^{\eta} C_{2} \lambda_{j}^{-\left(\sigma-\sigma_{0}\right)}\left(\widetilde{C} / 2 m_{j}\right)^{\sigma_{0}}\right)\right)\|U\|_{A_{J}}^{2} \\
&+C_{2} \lambda_{J}^{-\sigma}\left\|K_{J}^{m} U\right\|_{A_{J}}^{2} .
\end{aligned}
$$

To estimate the last term in (24), combine Lemma 2.1, (2.8), and (3.7). Hence,

$$
\lambda_{J}^{-\sigma}\left\|K_{J}^{m_{J}} U\right\|_{A_{J}}^{2} \leq\left(C_{3}^{\sigma_{0}}\right)^{\eta} \lambda_{J}^{-\left(\sigma-\sigma_{0}\right)}(\widetilde{C} / 2 m)^{\sigma_{0}}\|U\|_{A_{J}}^{2} .
$$


Finally, we combine $(25)$ with $(24)$ to conclude that $(3.16 a)$ holds with $\delta^{(2)}$ satisfying (22).

(3.16b) follows readily from (3.4) and (22). To prove (3.17a)-(3.17c), apply (3.16a) and Theorem 3.3.

Proof of Theorem 3.4N. Using (3.4) and (8), we have

$$
\tilde{\delta}_{J} \leq C_{2} \lambda_{J}^{-\sigma}\left(4+4 C_{2} \gamma^{\sigma}+\tilde{\delta}_{J-1}\left(3+2 C_{2} \gamma^{\sigma}\right)\right) \text {. }
$$

Since $C_{2}$ and $\gamma^{\sigma}$ are fixed constants, we may apply the induction argument used in the proof of $(3.16 \mathrm{a}, \mathrm{b})$ in Theorem $3.4 \mathrm{~S}$ with the right side of $(26)$ replacing the right side of $(20)$. This proves Theorem $3.4 \mathrm{~N}$.

\section{ACKNOWLEDGMENTS}

The author thanks Professor J. H. Bramble and Dr. J. E. Pasciak for several helpful discussions during the preparation of this manuscript. The author is also grateful to the referee for many useful comments and suggestions.

\section{BIBLIOGRAPHY}

1. A. Brandt, Multi-level adaptive solution to boundary value problems, Math. Comp. 31 (1977), 333-391.

2. W. Hackbusch, Multigrid methods and applications, Springer-Verlag, New York, 1985.

3. J. Mandel, S. McCormick, and R. Bank, Variational multigrid theory, Multigrid Methods (S. McCormick, ed.), SIAM, Philadelphia, 1986, pp. 131-178.

4. C. I. Goldstein, Analysis and applications of multigrid preconditioners for singularly perturbed boundary value problems, SIAM J. Numer. Anal. 26 (1989), 1090-1123.

5. N. H. Decker, The Fourier analysis of a multigrid preconditioner, Multigrid Methods, Proc. Third Copper Mountain Conference (S. McCormick, ed.), Marcel Dekker, New York, 1988, pp. 117-141.

6. D. Braess and W. Hackbusch, A new convergence proof for the multigrid method including the $\mathscr{V}$-cycle, SIAM J. Numer. Anal. 20 (1983), 967-975.

7. R. E. Bank and T. Dupont, An optimal order process for solving finite element equations, Math. Comp. 36 (1981), 35-51.

8. R. E. Bank and C. Douglas, Sharp estimates for multigrid rates of convergence with general smoothing and acceleration, SIAM J. Numer. Anal. 22 (1985), 617-633.

9. J. H. Bramble and J. E. Pasciak, New convergence estimates for multigrid algorithms, Math. Comp. 49 (1987), 311-330.

10. C. Douglas, Multigrid algorithms with applications to elliptic boundary value problems, SIAM J. Numer. Anal. 21 (1984), 236-254.

11. S. McCormick, Multigrid methods for variational problems: General theory for the $\mathscr{V}$ cycle, SIAM J. Numer. Anal. 22 (1985), 634-643.

12. N. H. Decker, J. Mandel, and S. V. Parter, On the role of regularity in multigrid methods, Multigrid Methods, Proc. Third Copper Mountain Conference (S. McCormick, ed.), Marcel Dekker, New York, 1988, pp. 143-156.

13. P. G. Ciarlet, The finite element method for elliptic problems, North-Holland, Amsterdam, 1978.

14. G. Strang and G. J. Fix, An analysis of the finite element method, Prentice-Hall, Englewood Cliffs, N.J., 1973. 
15. C. I. Goldstein, Variational crimes and error estimates in the finite element method, Math. Comp. 15 (1980), 1131-1157.

16. J. H. Bramble, J. E. Pasciak, and J. Xu, The analysis of multigrid algorithms with nonnested spaces or noninherited quadratic forms, Math. Comp. 56 (1991), 1-34.

17. S. C. Brenner, An optimal-order multigrid method for P1 nonconforming finite elements, Math. Comp. 52 (1989), 1-15.

18. H. C. Elman, Iterative methods for large, sparse, nonsymmetric systems of linear equations, Ph.D. Thesis, Yale Univ. Computer Science Dept., Report 229, 1981.

19. J. L. Lions and E. Magenes, Non-homogeneous boundary value problems and applications, Vol. I, Springer-Verlag, New York, 1972.

20. R. Scott, Interpolated boundary conditions in the finite element method, SIAM J. Numer. Anal. 12 (1975), 404-427.

Department of Applied Science, Brookhaven National Laboratory, Upton, New York 11973

E-mail address: goldstei@bnl.gov 